\title{
Cross Sectional and Longitudinal Fuzzy Clustering of the NUTS and Positioning of the Italian Regions with Respect to the Regional Competitiveness Index (RCI) Indicators with Contiguity Constraints
}

\author{
Pierpaolo D'Urso ${ }^{1} \cdot$ Livia De Giovanni ${ }^{2}$ (D) $\cdot$ Riccardo Massari ${ }^{1} \cdot$ Francesca G. M. Sica $^{3}$
}

Accepted: 17 May 2019 / Published online: 6 June 2019

(c) Springer Nature B.V. 2019

\begin{abstract}
In socio-economical clustering often the empirical information is represented by time-varying data generated by indicators observed over time on a set of subnational (regional) units. Usually among these units may exist contiguity relations, spatial but not only. In this paper we propose a fuzzy clustering model of multivariate time-varying data, the longitudinal fuzzy C-Medoids clustering with contiguity constraints. The temporal aspect is dealt with by using appropriate measures of dissimilarity between time trajectories. The contiguity among units is dealt with adding a contiguity matrix as a penalization term in the clustering model. The cross sectional fuzzy C-Medoids clustering with contiguity constraints is obtained considering one instant of time. The model is applied to the classification of the European NUTS on the basis of the observed dynamics of the Basic, Efficiency and Innovation subindexes of the Regional Competitiveness Index (RCI) 2013 and 2016. The positioning of the Italian regions is analyzed through the values of the medoids of the clusters and shows the peculiarities of the regions with respect to the subindexes either in single times or in the dynamic. Two contiguity constraints, one based on the European Western, Southern, Central and Northern geographic areas and one on the level of GDP - taken into account in the computation of the RCI-are also introduced in the models.
\end{abstract}

Keywords Regional Competitiveness Index (RCI) - European NUTS · Italian regions · Fuzzy clustering $\cdot$ Time trajectories $\cdot$ Contiguity constraints

Livia De Giovanni

ldegiovanni@luiss.it

Pierpaolo D'Urso

pierpaolo.durso@uniroma1.it

Riccardo Massari

riccardo.massari@uniroma1.it

Francesca G. M. Sica

f.sica@confindustria.it

1 Department of Social Sciences and Economics, Sapienza University of Rome, Rome, Italy

2 Department of Political Science, LUISS Guido Carli and CEFOP-LUISS, Rome, Italy

3 Economic Research Department, Confindustria and CEFOP-LUISS, Rome, Italy 


\section{Introduction}

In socio-economical clustering often the empirical information is represented by time-varying data generated by Composite Indexes (CIs) observed over time on a set of subnational (regional) units. Usually among these units may exist contiguity relations, spatial but not only.

Composite indexes (CIs) which compare country performance are increasingly recognised as a useful tool in policy analysis and public communication. The number of CIs in existence around the world is growing year after year (OECD 2008). Such composite indexes provide simple comparisons of countries that can be used to illustrate complex and sometimes elusive issues in wide-ranging fields, e.g., environment, economy, society or technological development. A composite index is formed when individual indicators are compiled into a single index on the basis of an underlying model. The composite index should ideally measure multi-dimensional concepts which cannot be captured by a single indicator, such as competitiveness, industrialisation, sustainability, single market integration, knowledge-based society. Recently multivariate sets of indicators have been proposed in the framework of the project "Beyond GDP". Born in 2007, the Beyond GDP initiative is about developing indicators that are as clear and appealing as GDP, but more inclusive of environmental and social aspects of progress (Mazziotta and Pareto 2018). Economic indicators such as GDP were never designed to be comprehensive measures of prosperity and well-being.

The OECD Framework for Measuring Well-Being and Progress is based on the recommendations made in 2009 by the Commission on the Measurement of Economic Performance and Social Progress to which the OECD contributed significantly. It also reflects earlier OECD work and various national initiatives in the field. This framework is built around three distinct domains: material conditions, quality of life and sustainability, each with their relevant dimensions.

New measures for assessing specific aspects as Quality of Government have been proposed (Charron et al. 2015).

The classification and positionig of the (geographic) units with respect to the indicators is generally developed using Cluster Analysis. When time information are available, the data are three-way data of type same units $\times$ same variables $\times$ time. Two relevant questions that arise are: $i$ ) the temporal analysis of single/composite indexes and $i i$ ) the presence of some relations among units, spatial but not only, as highlighted in Delgado-Marquez and García-Velasco (2018).

The main methodological ways in the literature to aggregate units characterised by similar behaviour across time are the model- feature-and observation based approach. Here we consider the observation-based approach (Caiado et al. 2015; D'Urso 2005; Coppi et al. 2010, and references therein). In the last decade, different fuzzy clustering algorithms have been proposed for both univariate and multivariate time varying-data (see, e.g., Coppi and D’Urso 2002, 2003, 2006; D’Urso et al. 2017a, b).

Similarly, different methods have been suggested in the clustering literature to discover spatial patterns for different kind of spatial units, e.g., urban areas or image pixels. The main challenge these methods overcome is the identification of a suitable algorithm to capture both spatial dependence and spatial heterogeneity. Following the categorisation suggested by Caiado et al. (2015), Fouedjio (2016) classifies clustering of spatial data into four main approaches: non-spatial clustering with geographical coordinates as additional variables; non-spatial clustering based on a spatial dissimilarity measure; spatially constrained 
clustering; model-based clustering. An example of spatially constrained fuzzy algorithm for urban areas is provided by Di Nola et al. (2000). Examples of applications for image pixels segmentation can be found in Chuang et al. (2006). An approach worth of notice consists in including a spatial penalty term in the objective function of the clustering method, as suggested by Pham (2001). While this proposal has been introduced for solving image segmentation problem, the idea beyond can be easily extended to the clustering of geographical areas (Coppi et al. 2010). In this paper more general contiguity relations among units are considered.

In this paper we introduce the Longitudinal Fuzzy C-Medoids Clustering with contiguity constraints. It is the Partitioning Around Medoid (PAM) version of the Longitudinal Fuzzy C-Means Clustering with contiguity constraints (Coppi et al. 2010). The temporal aspect is dealt with by using appropriate measures of dissimilarity between time trajectories. The contiguity among units is dealt with adding a contiguity matrix as a penalization term in the clustering model. The novelty with respect to existing Fuzzy C-Medoids Clustering models is the presence of multivariate time-varying data and of the "contiguity" constraints. The Cross Sectional Fuzzy C-Medoids Clustering with contiguity constraints is obtained considering one instant of time. Then it is a particular case of the longitudinal version.

The models are applied to the classification of the European NUTS on the basis of the observed dynamics of the Basic, Efficiency and Innovation subindexes of the Regional Competitiveness Index (RCI) 2013 and 2016. The positioning of the Italian regions is deeply analyzed. Two contiguity constraints, one based on the European Western, Southern, Central and Northern geographic areas and one on the level of GDP-taken into account in the computation of the RCI - are also introduced in the models. The classifications obtained with and without contraints are compared.

The paper is structured as follows: in Sect. 2 the RCI is presented; in Sect. 3 the Clustering models are introduced; in Sect. 4 the models are applied to the classification of the EU NUTS on the basis of the RCI. Sect. 5 concludes.

\section{Regional Competitiveness Index (RCI)}

The Regional Competitiveness Index (RCI) (Annoni and Dijkstra 2013, 2017; Annoni et al. 2017) is composed of 11 pillars that describe the different aspects of competitiveness. They are classified into three groups (subindexes): Basic, Efficiency and Innovation.

The Basic group (Table 1) includes five pillars: (1) Institutions; (2) Macroeconomic Stability; (3) Infrastructure; (4) Health; and (5) Basic Education. These represent the key basic drivers of all types of economies (Annoni and Dijkstra 2013; Annoni et al. 2017).

The Innovation group (Table 3) consists of three pillars: (9) Technological Readiness; (10) Business Sophistication; and (11) Innovation.

The complete list of all candidate indicators in RCI 2106 is provided in Tables 1, 2 and 3.

To correct for different range and measurement units, weighted z-scores are adopted with the regions' population sizes as weights. The RCI is obtained in steps: i) as an average of the indicators in each pillar, then as an ii) average of the pillars in each group Basic, Efficiency, Innovation, finally as an iii) average of the subindexes Basic, Efficiency and Innovation. Averages in i), ii) are simple averages, the RCI is obtained as a weighted average of the subindexes Basic, Efficiency and Innovation with weights depending on the stage 
Table 1 Indicators of the subindex Basic

\begin{tabular}{|c|c|c|}
\hline Sub-index & Pillar & Indicator \\
\hline Basic & Institutions & Corruption \\
\hline Basic & Institutions & Quality and accountability \\
\hline Basic & Institutions & Impartiality \\
\hline Basic & Institutions & Country level corruption perception \\
\hline Basic & Institutions & Regional level corruption perception \\
\hline Basic & Institutions & Voice and accountability \\
\hline Basic & Institutions & Political stability \\
\hline Basic & Institutions & Government effectiveness \\
\hline Basic & Institutions & Regulatory quality \\
\hline Basic & Institutions & Rule of law \\
\hline Basic & Institutions & Control of corruption \\
\hline Basic & Institutions & Ease of doing business \\
\hline Basic & Institutions & Property rights \\
\hline Basic & Institutions & Intellectual property protection \\
\hline Basic & Institutions & Efficiency of legal framework in settling disputes \\
\hline Basic & Institutions & Efficiency of legal framework in challenging regulations \\
\hline Basic & Institutions & Transparency of government policymaking \\
\hline Basic & Institutions & Business costs of crime and violence \\
\hline Basic & Institutions & Organized crime \\
\hline Basic & Institutions & Reliability of police services \\
\hline Basic & Macroeconomic Stability & Government deficit/surplus \\
\hline Basic & Macroeconomic Stability & National savings \\
\hline Basic & Macroeconomic Stability & Government bond yields \\
\hline Basic & Macroeconomic Stability & Government debt average \\
\hline Basic & Infrastructure & Motorway potential accessibility \\
\hline Basic & Infrastructure & Railway potential accessibility \\
\hline Basic & Infrastructure & Number of passenger flights \\
\hline Basic & Infrastructure & Intensity of high-speed railways \\
\hline Basic & Health & Road fatalities \\
\hline Basic & Health & Healthy life expectancy \\
\hline Basic & Health & Infant mortality \\
\hline Basic & Health & Cancer disease death rate \\
\hline Basic & Health & Heart disease death rate \\
\hline Basic & Health & Suicide death rate \\
\hline Basic & Basic Education & Share of low-achieving 15 years olds in reading \\
\hline Basic & Basic Education & Share of low-achieving 15 years olds in math \\
\hline Basic & Basic Education & Share of low-achieving 15 years olds in science \\
\hline
\end{tabular}

of development. The use of simple averages in the first two steps is based on the Principal Component Analysis (PCA), used to check for the internal consistency of each RCI pillar. Each pillar in a composite index describes a particular aspect of the latent phenomenon to be measured. As such aspects are not directly observable, they can only be measured by indicators which are assumed to be related to the aspect they describe and, hence, to 
Table 2 Indicators of the subindex Efficiency

\begin{tabular}{lll}
\hline Sub-index & Pillar & Indicator \\
\hline Efficiency & Higher Education & Population 25-64 with higher educational attainment, \% \\
Efficiency & Higher Education & Participation of adults 25-64 in education and training, \% \\
Efficiency & Higher Education & People with at most lower secondary education, \% of 18-24 \\
Efficiency & Labor Market Efficiency & Employment rate (excluding agriculture) \\
Efficiency & Labor Market Efficiency & Long-term unemployment \\
Efficiency & Labor Market Efficiency & Unemployment rate \\
Efficiency & Labor Market Efficiency & Labour productivity \\
Efficiency & Labor Market Efficiency & Gender balance of unemployment \\
Efficiency & Labor Market Efficiency & Gender balance of employment \\
Efficiency & Labor Market Efficiency & Female unemployment \\
Efficiency & Labor Market Efficiency & Share 15-24 not in education, employment, training (NEET) \\
Efficiency & Market Size & Disposable income per capita \\
Efficiency & Market Size & Potential market size expressed in GDP (PPS) \\
Efficiency & Market Size & Potential market size expressed in population \\
\hline
\end{tabular}

Table 3 Indicators of the subindex Innovation

\begin{tabular}{|c|c|c|}
\hline Sub-index & Pillar & Indicator \\
\hline Innovation & Technological Readiness & Households with access to broadband \\
\hline Innovation & Technological Readiness & Individuals buying over Iinternet \\
\hline Innovation & Technological Readiness & Household access to internet \\
\hline Innovation & Technological Readiness & Availability of latest technologies \\
\hline Innovation & Technological Readiness & Firm-level technology absorption \\
\hline Innovation & Technological Readiness & Technological adoption \\
\hline Innovation & Technological Readiness & FDI and technology transfer \\
\hline Innovation & Technological Readiness & Enterprises having purchased online (at least 1\%) \\
\hline Innovation & Technological Readiness & Enterprises having received orders online (at least 1\%) \\
\hline Innovation & Technological Readiness & Enterprises with fixed broadband access \\
\hline Innovation & Business Sophistication & Employment (K-N sectors) \\
\hline Innovation & Business Sophistication & GVA (K-N sectors) \\
\hline Innovation & Business Sophistication & Innovative SMEs collaborating with others \\
\hline Innovation & Innovation & Total patent applications \\
\hline Innovation & Innovation & Core creative class employment \\
\hline Innovation & Innovation & Knowledge workers \\
\hline Innovation & Innovation & Scientific publications \\
\hline Innovation & Innovation & Human Resources in Science and Technology (HRST) \\
\hline Innovation & Innovation & Total intramural R\&D expenditure \\
\hline Innovation & Innovation & Employment in technology and knowledge-intensive average \\
\hline Innovation & Innovation & High-tech patents \\
\hline Innovation & Innovation & ICT patents \\
\hline Innovation & Innovation & Exports in medium-high/high tech manufacturing \\
\hline
\end{tabular}


Table 4 GDP levels and weights RCI 2016

\begin{tabular}{llll}
\hline Stage of development & Basic & Efficiency & Innovation \\
\hline Stage $1<50$ & 35.00 & 50.00 & 15.00 \\
Stage 2 50-75 & 31.25 & 50.00 & 18.75 \\
Stage 3 75-90 & 27.50 & 50.00 & 22.50 \\
Stage 4 90-110 & 23.75 & 50.00 & 26.25 \\
Stage 5 $>110$ & 20.00 & 50.00 & 30.00 \\
\hline
\end{tabular}

each other.The PCA for each pillar determines the list of artificial variable(s) named principal components each containing all the indicators of the pillar with different weights each accounting for as much of the variability in the data as possible in decreasing order. The conditions to be verified to use only one pillar-obtained as a simple average of the indicators measuring that pillar-are that each pillar shows a unique, most relevant PCA accounting for a large amount of variance and that all the indicators contribute to roughly the same extent to the first principal component. To obtain the RCI as a weighted average of the three subindexes EU regions are divided into five development stages based on their average 2012-2014 GDP per head in purchasing power standard (PPS) expressed as an index (EU-28 = 100). The five development stages and the percentage weights of the subindexes are defined for the year 2016 according to Table 4.

\subsection{RCI Pillars and Subpillars 2016 and 2013: Main Results}

The value of RCI 2016 and of the three subindexes Basic, Efficiency, Innovation for all the NUTS are in Table 5. The value of the RCI in 2013 and 2016, the values of the three subindexes Basic, Efficiency, Innovation in 2013 and 2016 and the values of the eleven Pillars for the Italian regions are described in Table 6.

In Table 7 the RCI 2013 and 2016 in decreasing order for the Italian regions are presented. In Table 8 the detail of the 3 subindexes and the relative absolute and percentual variations 2013-2016 is presented. Italy has 21 NUTS2 regions. They are grouped into 5 NUTS1 levels:

- NORD-OVEST: Piemonte (ITC1), Valle d'Aosta (ITC2), Liguria (ITC3), Lombardia (ITC4)

- NORD-EST: Bolzano (ITH1), Trento (ITH2), Veneto (ITH3), Emilia Romagna (ITH5), Friuli Venezia Giulia (ITH4)

- CENTRO: Toscana (ITI1), Umbria (ITI2), Marche (ITI3), Lazio (ITI4)

- SUD: Abruzzo (ITF1), Molise (ITF2), Campania (ITF3), Puglia (ITF4), Basilicata (ITF5), Calabria (ITF6)

- ISOLE: Sicilia (ITG1), Sardegna (ITG2).

Table 9 lists scores and ranks for each member of the European Union. The final score at country level is computed as regional population weighted average. In 2016 Italy ranks 18th in EU 28 and slips two spots compared to 2013 (16th in 2010): its score has deteriorated from -0.40 in 2013 to -0.48 (it was -0.30 in 2010). Italy is emerging from a long and deep recession but a stagnating economy since the late of 1990s has left the economy 


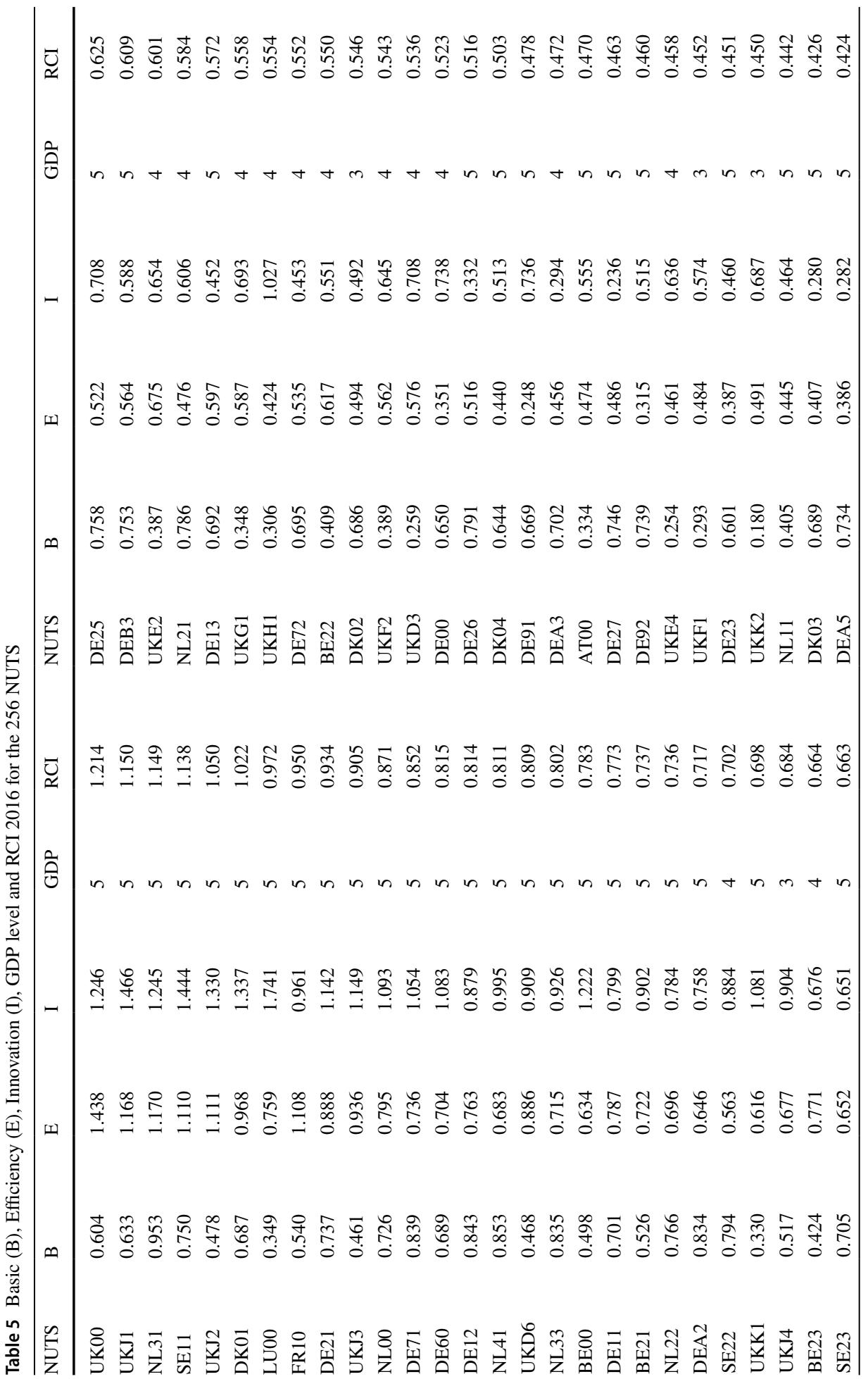




\begin{tabular}{|c|c|c|c|}
\hline$\vec{\circlearrowright}$ & 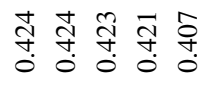 & 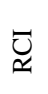 & 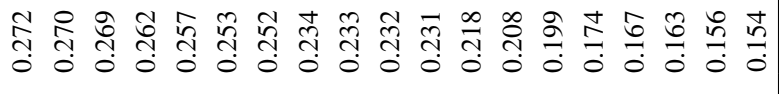 \\
\hline วิ & $\theta \ln n t$ & Оิ & $m n n+n m n n+m n+t m+m+m$ \\
\hline- & 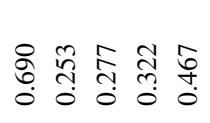 & - & 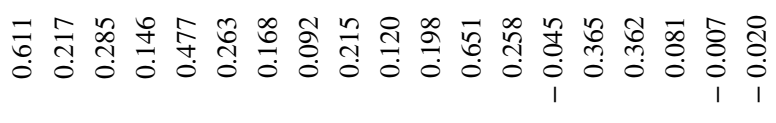 \\
\hline 山ు & 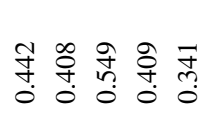 & 띠 & 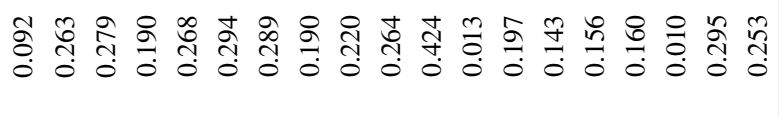 \\
\hline 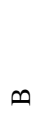 & 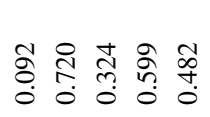 & م & 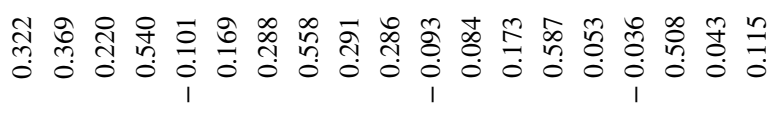 \\
\hline$\stackrel{n}{5}$ & 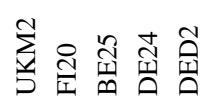 & $\stackrel{n}{5}$ & 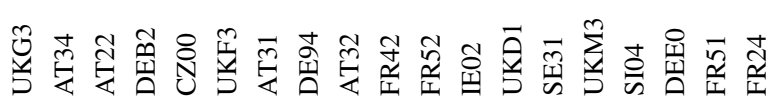 \\
\hline 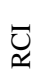 & 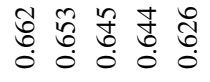 & $\ddot{\mho}$ & 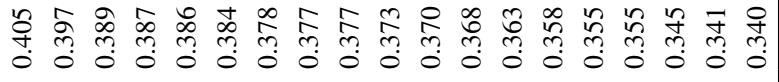 \\
\hline ชิ & $n n t \forall n$ & Оิ & 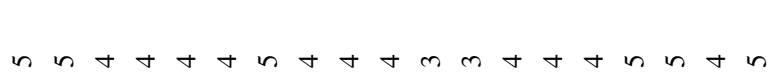 \\
\hline - & $\begin{array}{lllll}0 & 0 & 0 & 0 \\
n & 0 & 0 & 0 & 0 \\
0 & 0 & 0 & 0 & 0 \\
0 & 0 & 0 & 0\end{array}$ & 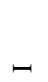 & 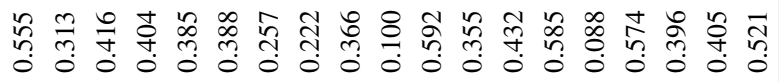 \\
\hline 피 & 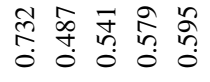 & 띠 & 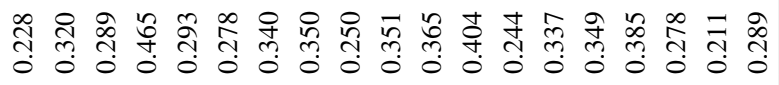 \\
\hline 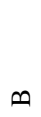 & 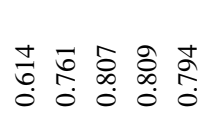 & $\infty$ & 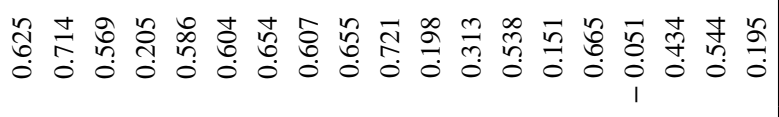 \\
\hline 5 & 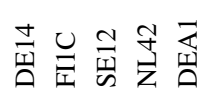 & $\overbrace{5}^{n}$ & 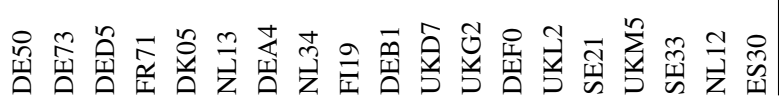 \\
\hline
\end{tabular}




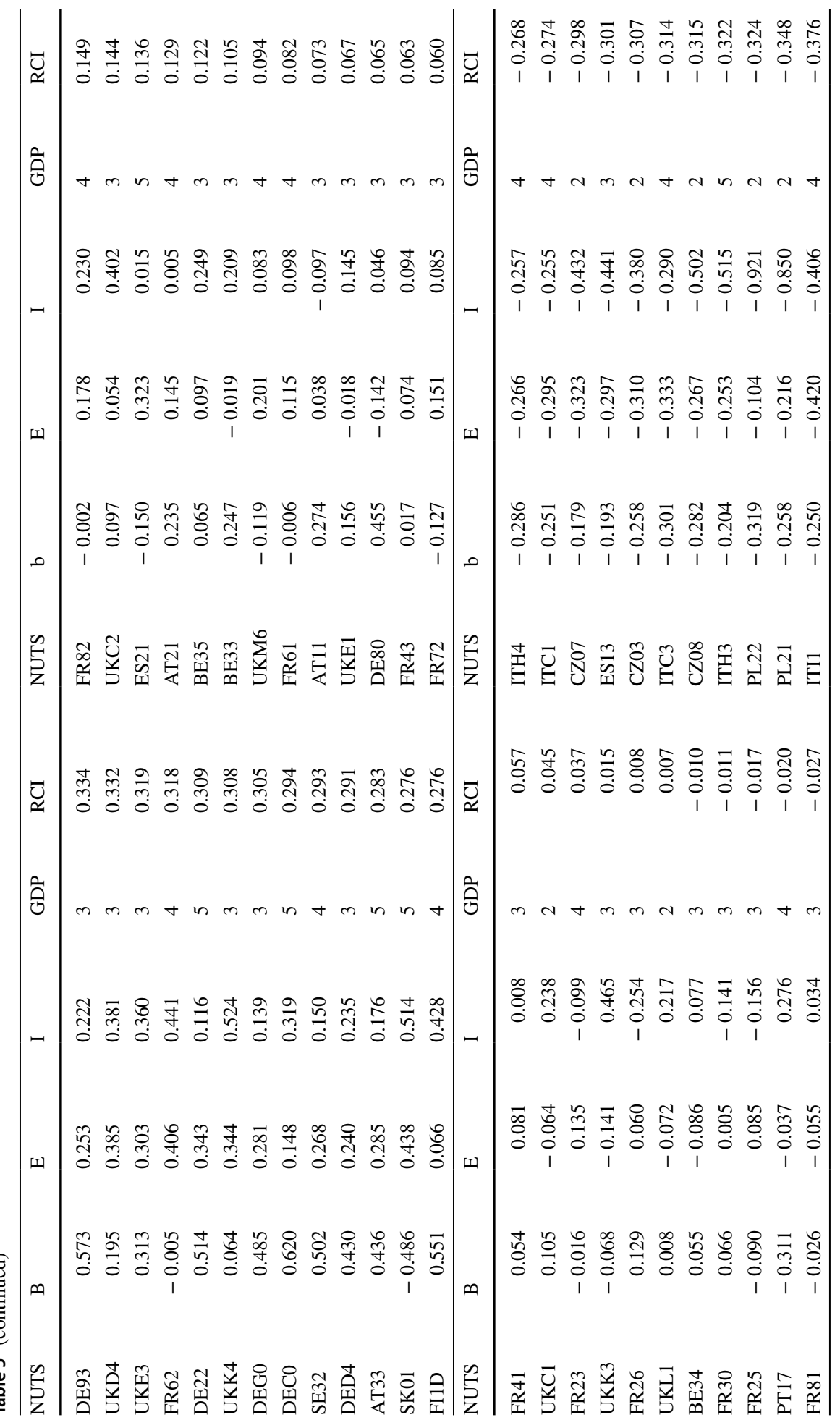




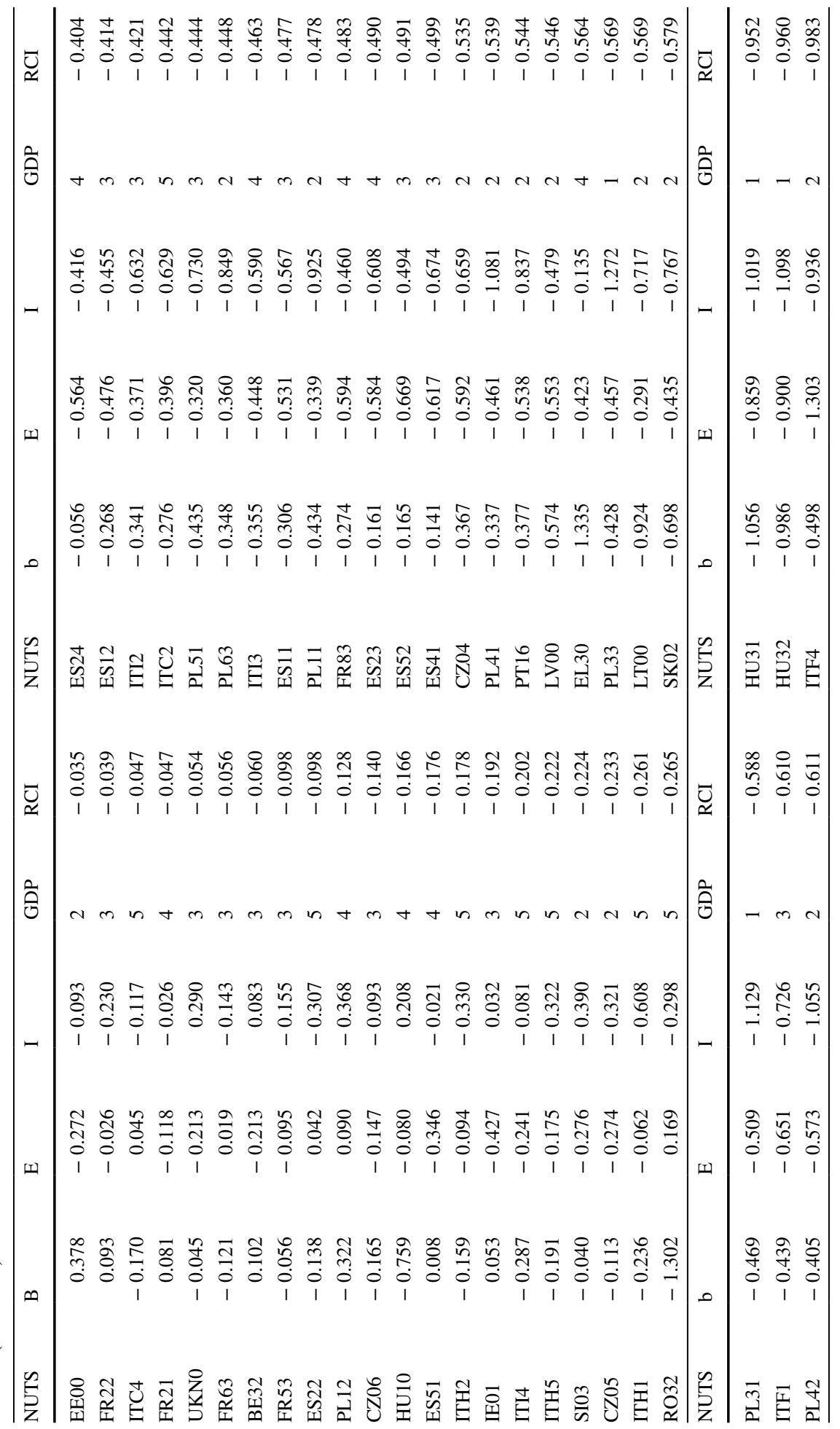




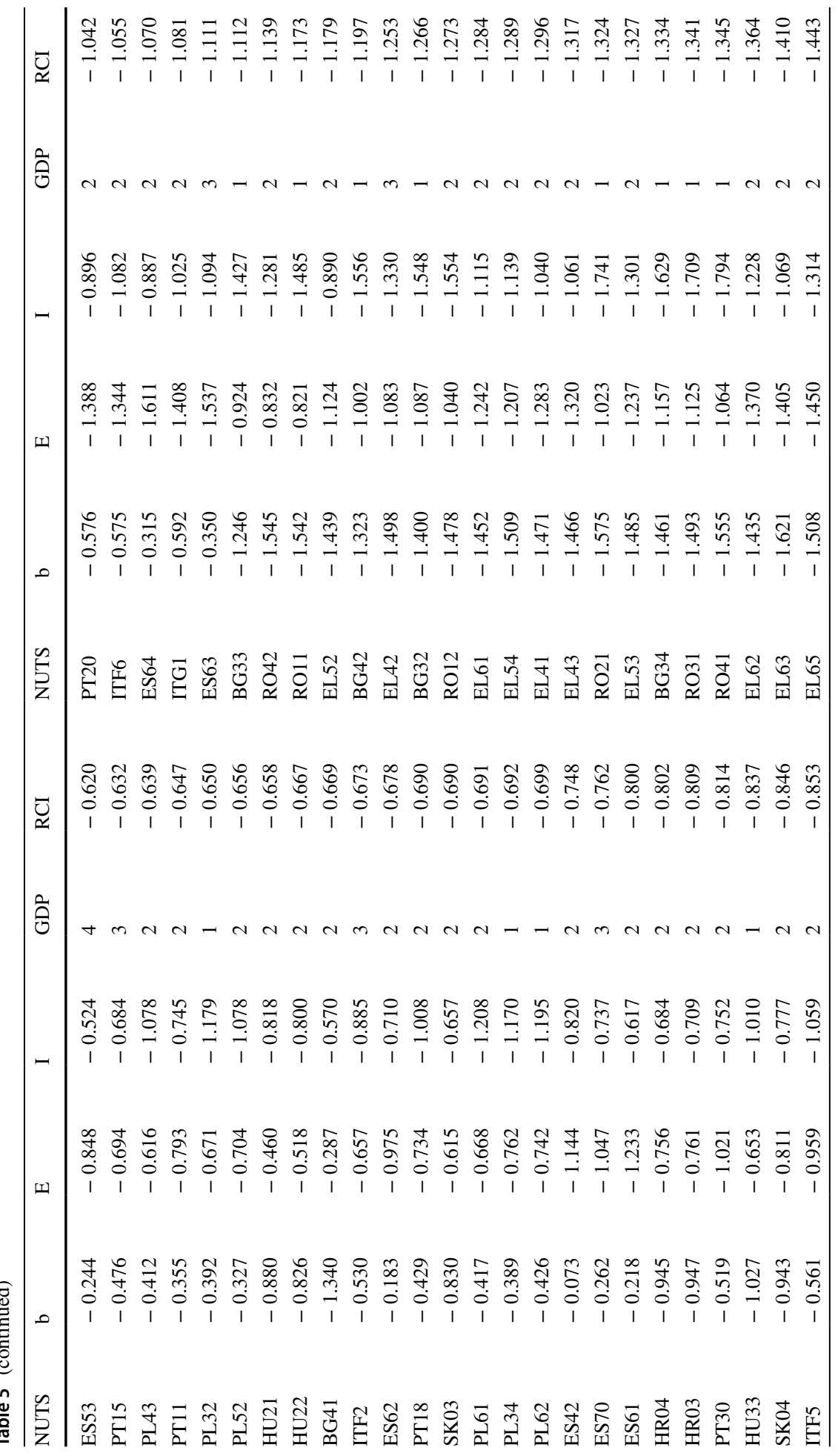




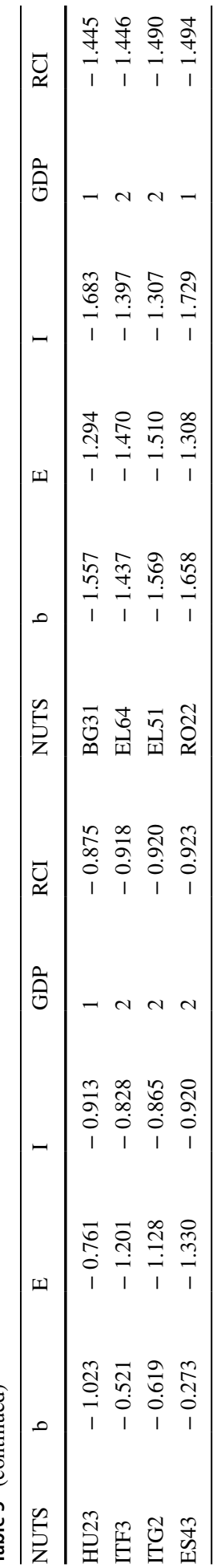




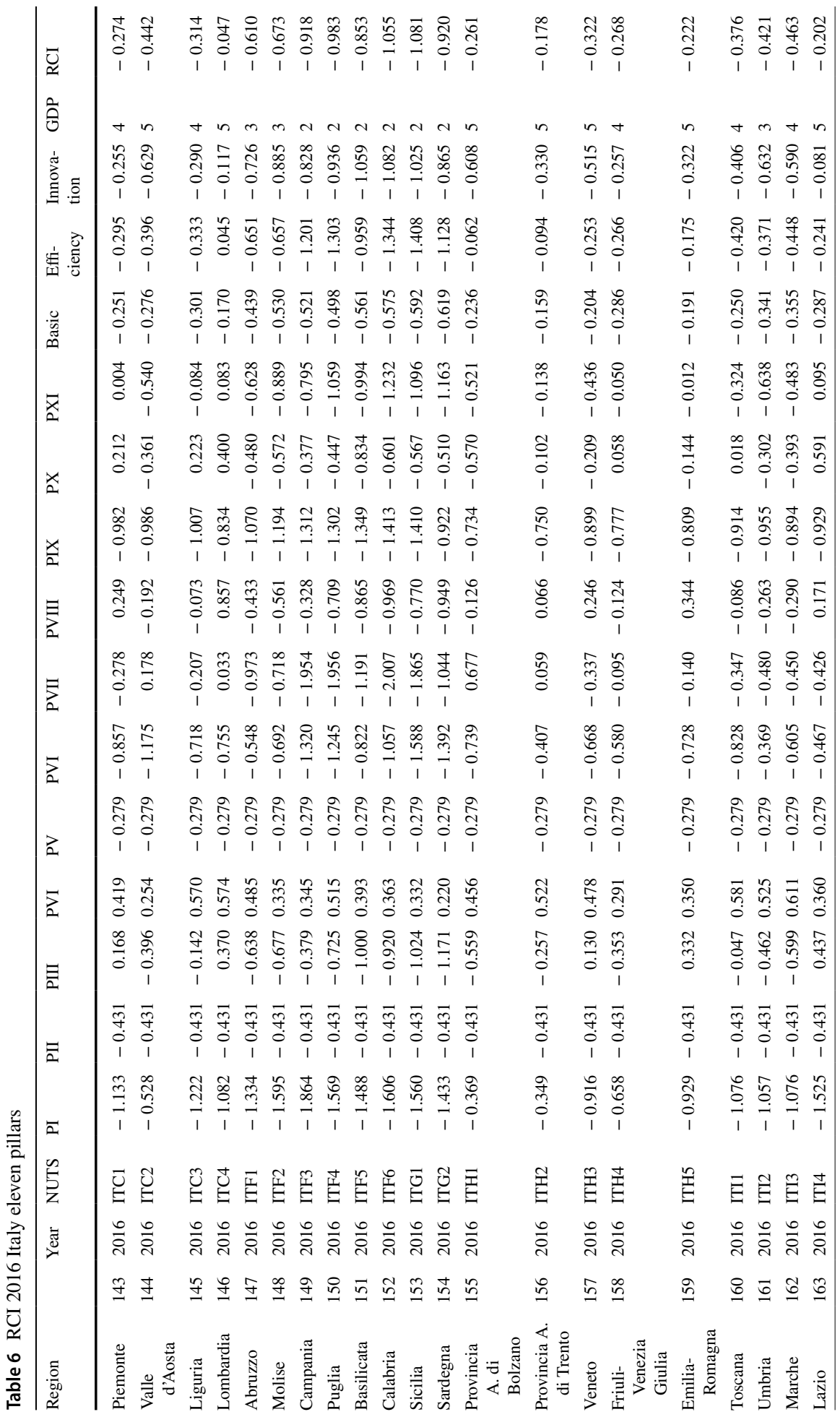




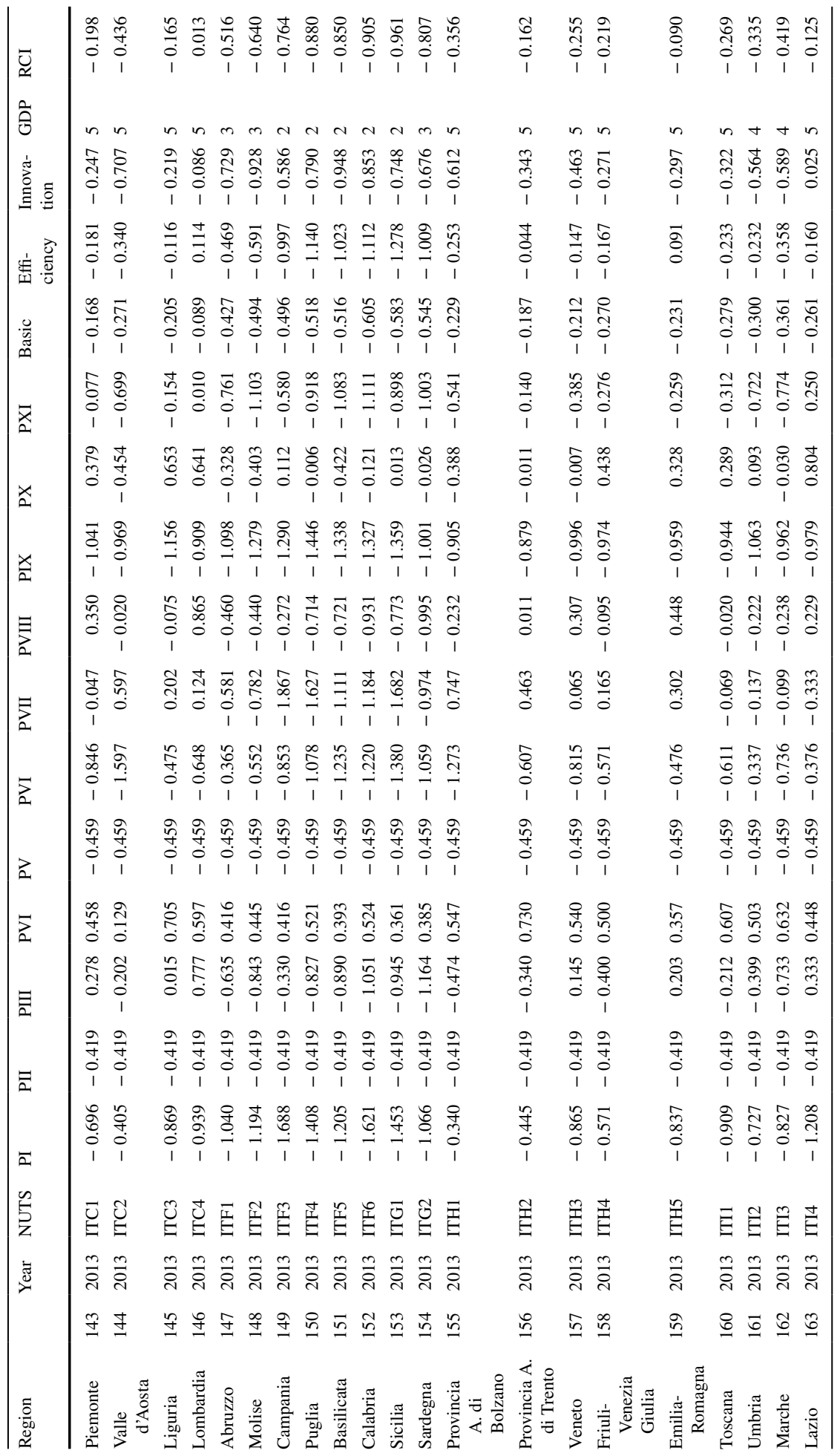


Table 7 RCI 2013 and 2016 for Italian regions (z-scores)

\begin{tabular}{llrlll}
\hline Region & NUTS & RCI 2013 & Region & NUTS & RCI 2016 \\
\hline Lombardia & ITC4 & 0.013 & Lombardia & ITC4 & -0.047 \\
Emilia-Romagna & ITH5 & -0.090 & Provincia Autonoma di Trento & ITH2 & -0.178 \\
Lazio & ITI4 & -0.125 & Lazio & ITI4 & -0.202 \\
Provincia Autonoma di Trento & ITH2 & -0.162 & Emilia-Romagna & ITH5 & -0.222 \\
Liguria & ITC3 & -0.165 & Provincia Autonoma di Bolzano & ITH1 & -0.261 \\
Piemonte & ITC1 & -0.198 & Friuli-Venezia Giulia & ITH4 & -0.268 \\
Friuli-Venezia Giulia & ITH4 & -0.219 & Piemonte & ITC1 & -0.274 \\
Veneto & ITH3 & -0.255 & Liguria & ITC3 & -0.314 \\
Toscana & ITI1 & -0.269 & Veneto & ITH3 & -0.322 \\
Umbria & ITI2 & -0.335 & Toscana & ITI1 & -0.376 \\
Provincia Autonoma di Bolzano & ITH1 & -0.356 & Umbria & ITI2 & -0.421 \\
Marche & ITI3 & -0.419 & Valle d'Aosta/Vallée d'Aoste & ITC2 & -0.442 \\
Valle d'Aosta/Valle d'Aoste & ITC2 & -0.436 & Marche & ITI3 & -0.463 \\
Abruzzo & ITF1 & -0.516 & Abruzzo & ITF1 & -0.610 \\
Molise & ITF2 & -0.640 & Molise & ITF2 & -0.673 \\
Campania & ITF3 & -0.764 & Basilicata & ITF5 & -0.853 \\
Sardegna & ITG2 & -0.807 & Campania & ITF3 & -0.918 \\
Basilicata & ITF5 & -0.850 & Sardegna & ITG2 & -0.920 \\
Puglia & ITF4 & -0.880 & Puglia & ITF4 & -0.983 \\
Calabria & ITF6 & -0.905 & Calabria & ITF6 & -1.055 \\
Sicilia & ITG1 & -0.961 & Sicilia & ITG1 & -1.081 \\
\hline
\end{tabular}

behind in many dimensions of RCI: institutions, human capital and labour market continue to be its weakest areas.

The Italian business environment hinders private investment for a number of reasons, including an inefficient legal framework, high taxation and regulations that disincentives Foreign Direct Investments (WEF 2017). Reforms implemented in recent years have improved population perception of corruption. In particular the New Italian Public Procurement Code strengthens the role of ANAC, the National Anti-bribery and Corruption Authority and updates award criteria to include preference based on the "most economically advantageous tender". But nevertheless the public sector performance remains poor with a highly inefficient judicial system: the time needed to resolve civil, commercial, administrative and other cases is 395 days for the 1 st instance compared to 154 in Romania, 17 in Denmark. The Italian labour market has become more efficient in 2015 with the adoption of the "Jobs Act" which introduced three major novelties to Italy's employment protection legislation: (1). it allows temporary contracts to be renewed up to 8 times (from 5) within a maximum overall duration of 36 months and abolishes the obligation to express the rationale of the temporary contract; (2). it overhauls Art. 18 of Workers' Charter, already modified in 2012 by the Fornero reform, which regulates dismissals in firms with more than 15 employees (European Commission 2017); (3). it restricts the use of atypical contracts. But nevertheless worker's skills are deficient as captured by the OECD Programme for the International Assessment of Adult Competencies (PIAAC OECD 2016: the mean proficiency score of 16-65 year-olds in literacy 250.5 and in numeracy 247.1 


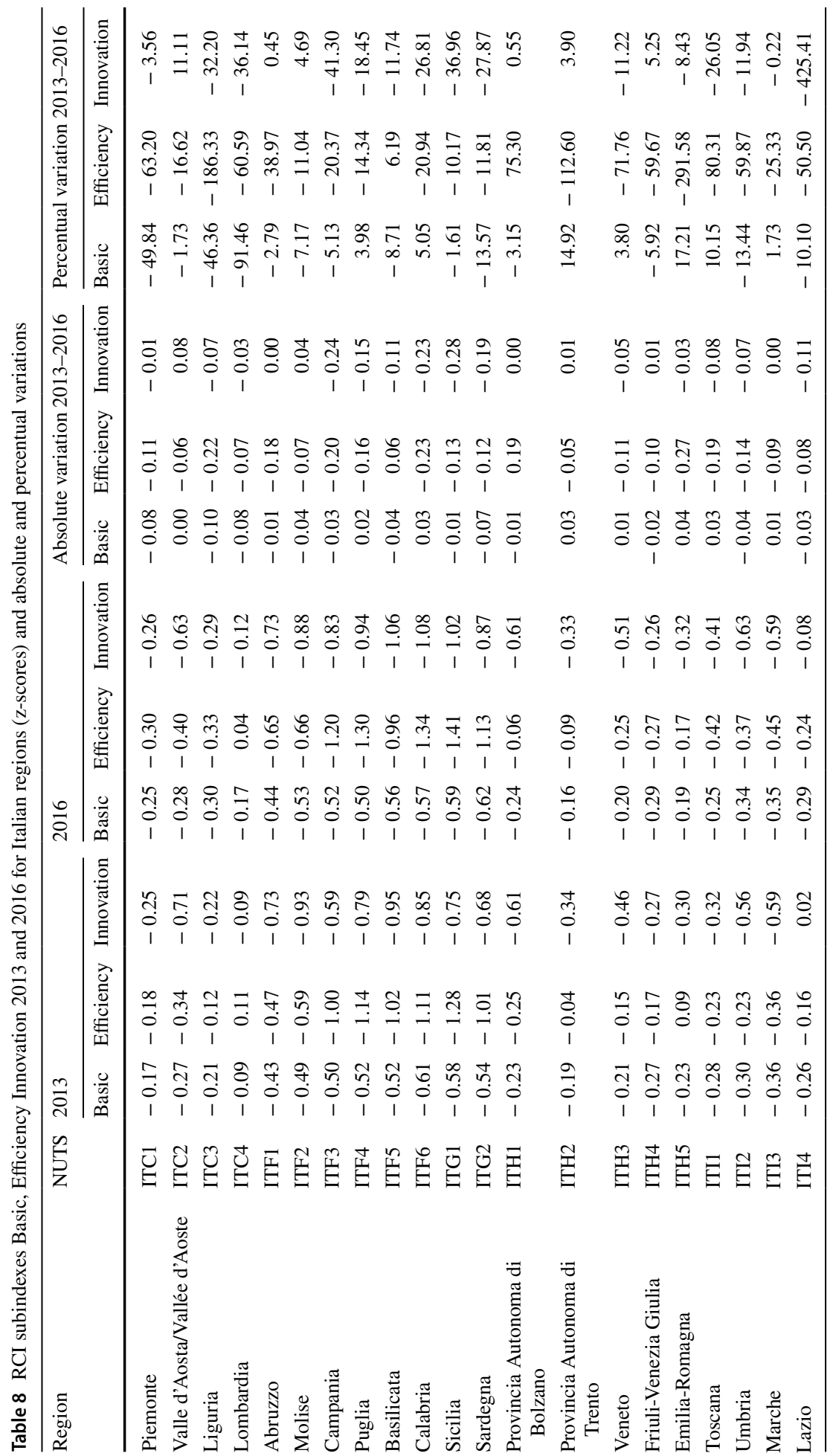


Table 9 RCI regional population weighted average, regional minimum and maximum

\begin{tabular}{|c|c|c|c|c|}
\hline 1 & Luxembourg & 0.97 & & \\
\hline 2 & Netherlands & 0.76 & 0.34 & 1.15 \\
\hline 3 & Sweden & 0.68 & 0.20 & 1.14 \\
\hline 4 & Denmark & 0.64 & 0.39 & 1.02 \\
\hline 5 & United Kingdom & 0.60 & 0.05 & 0.54 \\
\hline 6 & Germany & 0.54 & 0.06 & 0.93 \\
\hline 7 & Finland & 0.51 & 0.28 & 0.90 \\
\hline 8 & Belgium & 0.49 & -0.06 & 0.78 \\
\hline 9 & Austria & 0.33 & 0.07 & 0.47 \\
\hline 10 & France & 0.25 & -1.50 & 0.95 \\
\hline 11 & Ireland & 0.01 & -0.19 & 0.22 \\
\hline 12 & Estonia & -0.04 & & \\
\hline 13 & Czechia & -0.16 & -0.53 & 0.26 \\
\hline 14 & Slovenia & -0.22 & -0.22 & 0.17 \\
\hline 15 & Spain & -0.39 & -1.11 & 0.34 \\
\hline 16 & Poland & -0.47 & -0.70 & -0.13 \\
\hline 17 & Portugal & -0.47 & -1.04 & -0.02 \\
\hline 18 & Italy & -0.48 & -1.08 & -0.05 \\
\hline 19 & Cyprus & -0.49 & & \\
\hline 20 & Malta & -0.50 & & \\
\hline 21 & Hungary & -0.52 & -0.96 & -0.17 \\
\hline 22 & Latvia & -0.55 & & \\
\hline 23 & Lithuania & -0.57 & & \\
\hline 24 & Slovakia & -0.59 & -0.85 & 0.28 \\
\hline 25 & Croatia & -0.81 & -0.81 & -0.80 \\
\hline 26 & Greece & -1.05 & -1.49 & -0.56 \\
\hline 27 & Bulgaria & -1.08 & -1.44 & -0.67 \\
\hline 28 & Romania & -1.18 & -1.49 & -0.27 \\
\hline
\end{tabular}

are significantly below the average of the 29 OECD countries participating in the Survey of Adult Skills (PIAAC (267.7 and 263.0 respectively). Beside the ultra-Broadband Plan 2020 with a target of $85 \%$ of population covered (OECD 2017), in 2016 the government launched the National Industry 4.0 Plan (ISTAT 2018) ${ }^{1}$, the first national industry plan explicitly aiming at modernising the productive structure of the economy by providing a

\footnotetext{
${ }^{1}$ The key incentives to boost investment include:-Hyper-depreciation scheme (introduced with the budget law of 2017): companies will be allowed to deduct 250-Super-depreciation (introduced in 2016 and enhanced in 2017): companies will be allowed to deduct from their taxable income a sum equal to $140 \%$ of the original cost of eligible equipment, machineries, software (if connected to investments in industry 4.0 technologies) and other eligible equipment; - Strengthened R\&D tax credits for 2017 by raising the share of internal $\mathrm{R} \& \mathrm{D}$ spending that is deductible from companies' taxable income to $50 \%$ (from $25 \%$ ) - the same as for external R\&D spending_-and raising the annual tax-credit celling to EUR 20 million (from EUR 5 million); Stronger incentives for investing in start-ups and innovative SMEs by: raising the tax credit to $30 \%$ (from 19\%) of the invested capital in start-ups and innovative SMEs and raising the maximum eligible investment to EUR 1 million (from EUR 0.5 million); allowing companies to claim a tax credit equivalent to losses of controlled start-ups for the first four years of activity; boosting venture capital dedicated to selected industry 4.0 technologies through co-investment schemes with private sector funds.
} 
range of incentives (for about EUR 13 billion) to boost innovation and skills in new technologies over 2017-2020. The Industry 4.0 Plan also aims at enhancing the supply of skills relating to new technology by: implementing the Digital School National Plan; increasing the number of students (at university and post-secondary vocational and education training courses) and doctoral researchers in technical and scientific subjects; creating competence centres and digital innovation hubs to promote cooperation and exchanges among universities, large companies and SMEs, start-ups, business associations and public sector, aiming at supporting the technological transfer and enhancing technical and managerial skills on new technologies.

The distribution of scores across regions is also shown in Table 9: countries are sorted from largest to smallest in terms of internal dispersion measured by the range of variation i.e. the difference between the highest and the lowest score at regional level. France has the largest regional disparities among the European countries, while Italy among the most advanced members is the only one that exhibits negative scores across all its regions, including its capital region (Lazio ITI4 - 0.202): the maximum score is -0.047 (Lombardia ITC4), the minimum score is -1.081 (Sicilia ITG1).

\section{Cross Sectional and Longitudinal Fuzzy Clustering with Contiguity Constraints}

By considering an exploratory approach, we analyze the three-way data array of type same units $\times$ same quantitative variables $\times$ time. This type of three-way data array is called time data array. A time data array can be algebraically formalized as follows: $\mathbf{X} \equiv\left\{x_{i j t}, i=1 ; \ldots, I, j=1, \ldots, J ; t=1, \ldots, T\right\}$ where $i(i=1, \ldots, I)$ indicates the unit, $j(j=1, \ldots, J)$ the variable, and $t(t=1, \ldots, T)$ the time. Then, the generic element of $\mathbf{X}, x_{i j t}$, represents the $j$-th variable observed on the $i$-th spatial unit at time $t$. We can denote $\mathbf{X}$ also in the following way: $\mathbf{X} \equiv\left\{\mathbf{x}_{i}, i=1 ; \ldots, I\right\}$, where $\mathbf{x}_{i}=\left\{x_{i 11}, \ldots, x_{i J T}\right\}$. The time data array $\mathbf{X}$ can be represented by a bi-dimensional matrix by combining two of the three indices $i, j, t$ on the rows and assigning the remaining index to the columns. In this paper, we analyse only the case in which the time data array $\mathbf{X}$ is represented in the space of the units $\Re^{J+1}$ (the first $J$ dimensions correspond to the $J$ variables and the last dimension is referred to the time). In this space, each unit $i$ is represented, for each time $t$, by the vector $\mathbf{x}_{i t}=\left\{x_{i 1 t}, \ldots, x_{i J t}\right\}$ (D'Urso 2004, 2005).

In order to suitably incorporate constraints in the clustering procedure, a squared matrix of order $I$, the contiguity matrix $\mathbf{P}$, is introduced. It might be a spatial matrix of adjacency constraints, in case of spatial relations, or a matrix incorporating other contiguity relations among units to be taken into account in the clustering procedure. Notice that the diagonal elements of $\mathbf{P}$ are conventionally set equal to zero in order to allow the algebraic manipulation of $\mathbf{P}$.

\subsection{Cross Sectional and Longitudinal Fuzzy C-Medoids Clustering with Contiguity Constraints}

Fuzzy clustering is an overlapping approach which allows cases to belong to more than one cluster simultaneously as opposed to crisp clustering which results in mutually exclusive clusters (Bezdek 1981). In particular, in crisp clustering "each datum is exactly assigned to only one cluster obtaining exhaustive partitions characterized by nonempty and pairwise disjoint 
subsets. Such crisp assignment of data to clusters can be inadequate in presence of data points that are almost equally distant from two or more clusters. Such special data points can represent hybrid-type or mixture objects, which are (more or less) equally similar to two or more types. A crisp partition arbitrarily forces the full assignment of such data points to one of the clusters, although they should (almost) equally belong to all of them. Fuzzy clustering relaxes the requirement that data points have to be assigned to one (and only one) cluster. Data points can belong to more than one cluster and even with different degrees of membership to the different clusters. This gradual cluster assignment can reflect cluster structure in a more natural way, especially when clusters overlap. Then, the memberships of data points at the overlapping boundaries can express the ambiguity of the cluster assignment (Kruse et al. 2007)". The principal advantages connected to the fuzzy approach are the following (Hwang et al. 2007): (1) Due to the difficulty of identifying a clear boundary between clusters in real applications, fuzzy clustering appears more attractive than the crisp (non-fuzzy) clustering methods (McBratney and Moore 1985; Wedel and Kamakura 1998). (2) The memberships indicate whether there is a second-best cluster almost as good as the best cluster, a scenario which hard clustering methods cannot uncover (Everitt et al. 2001). (3) The fuzzy clustering is attractive because it is easily compatible with distribution free methods. (4) The fuzzy clustering is computationally efficient (McBratney and Moore 1985; Heiser and Groenen 1997).

There are several real cases in which it is more suitable to identify prototypes belonging to the considered dataset, that synthesize the structural information of each cluster, the so-called medoids. Several clustering techniques based on medoids have been proposed, e.g., the Partitioning Around Medoids (PAM) proposed by Kaufman and Rousseeuw (2005). In a fuzzy framework, Krishnapuram et al. (1999) Krishnapuram et al. (2001) suggested the so-called Fuzzy C-Medoids clustering method. Notice that using the Partitioning Around Medoids (PAM) approach, the prototypes of each cluster, henceforth medoids, are regions actually observed and not "virtual" regions like the "centroids" derived with a fuzzy C-means clustering approach. Overall, having non-fictitious representative regions available makes interpreting the obtained clusters easier, which is often very useful in geographical applications. In fact, "in many clustering problems one is particularly interested in a characterization of the clusters by means of typical or representative objects [regions]. These are objects [regions] that represent the various structural aspects of the set of objects [regions] being investigated. There can be many reasons for searching for representative objects [regions]. Not only can these objects [regions] provide a characterization of the clusters, but they can often be used for further work or research, especially when it is more economical or convenient to use a small set of $k$ objects [regions] instead of the large set one started off with" (Kaufman and Rousseeuw 2009). We observe that PAM-based fuzzy clustering represents a robustification of the fuzzy C-means clustering; however, it provides only a "timid robustification" of the fuzzy C-means clustering, because a single outlier still serves to breakdown the clustering (GarcíaEscudero and Gordaliza 1999). As remarked by García-Escudero et al. García-Escudero et al. (2010) the clustering based on medoids "resists to the presence of 1 outlier in a remote position, but it breaks down when we increase to 3 the number of outliers".

In this section the Longitudinal Fuzzy C-Medoids Clustering model with Contiguity constraints (L-FCMd-C) is introduced. It is a Partition Around Medoids (PAM) version of the model suggested by Coppi et al. (2010). The L-FCMd-C model is formalized in the following way:

$$
\min : \quad \sum_{i=1}^{I} \sum_{c=1}^{C} u_{i c}^{m} \sum_{t=1}^{T}\left(w_{t} d\left(\mathbf{x}_{i t}, \tilde{\mathbf{x}}_{c t}\right)\right)^{2}+\frac{\beta}{2} \sum_{i=1}^{I} \sum_{c=1}^{C} u_{i c}^{m} \sum_{i^{\prime}=1}^{I} \sum_{c^{\prime} \in C_{c}} p_{i i^{\prime}} u_{i^{\prime} c^{\prime}}^{m}
$$




$$
\begin{aligned}
& \sum_{t=1}^{T} w_{t}=1, w_{t} \geq 0 \\
& \sum_{c=1}^{C} u_{i c}=1, u_{i c} \geq 0
\end{aligned}
$$

where $u_{i c}$ denotes the membership degree of the $i$-th unit to the $c$-th cluster; $d\left(\mathbf{x}_{\mathbf{i t}}, \tilde{\mathbf{x}}_{\mathbf{c t}}\right)$ is a suitable Euclidean distance between the $i$-th unit $\mathbf{x}_{i t}=\left\{x_{i 1 t}, \ldots, x_{i J t}\right\}$ and the medoid of the $c$-th cluster $\tilde{\mathbf{x}}_{c t}=\left\{\tilde{x}_{c 1 t}, \ldots, \tilde{x}_{c J t}\right\}$ at time $t, m>1$ is a parameter controlling the fuzziness of the partition (for the selection of $m$, see D'Urso 2015), $\mathbf{P} \equiv\left\{p_{i i^{\prime}}, i=1 ; \ldots, I, i^{\prime}=1 ; \ldots, I\right\}$ is the contiguity matrix ( $p_{i i^{\prime}}=1$ for contiguous $i, i^{\prime}$; 0 otherwise), $w_{t}$ is the tuning parameter of the temporal information; $\beta \geq 0$ is the tuning parameter of the spatial information; $C$ is the number of clusters.

The objective function (1) is optimized with respect to the medoids $\tilde{\mathbf{x}}_{c}(c=1, \ldots C)$, chosen among the $n$ units, the membership degrees $u_{i c}$ and the temporal weights $w_{t}$. The medoids corresponding to the optimization solution provide a fuzzy partition via $u_{i c}$.

The objective function cannot be minimized by means of the alternating optimization algorithm, because the necessary conditions cannot be derived by differentiating it with respect to the medoids. Nonetheless, following heuristic algorithm of Fu (1982) for a crisp version (corresponding to $m=1, u_{i c}=1$ or 0 ) of the objective function, a fuzzy clustering algorithm that minimizes the objective function can be built up (Krishnapuram et al. 2001).

As far as the Euclidean distance $d\left(\mathbf{x}_{i t}, \tilde{\mathbf{x}}_{c t}\right)$ is concerned, two types of dissimilarity measures for multivariate trajectories are used (Coppi and D’Urso 2001, 2006; D’Urso 2004, 2005): the dissimilarity that compares the time trajectories for the different time instants, i.e. $d\left(\mathbf{x}_{i t}, \tilde{\mathbf{x}}_{c t}\right)=\left\|\mathbf{x}_{i t}-\tilde{\mathbf{x}}_{c t}\right\|, t=1, \ldots, T$ and the dissimilarity that considers the variation $\mathbf{v}_{i t}=\left(\mathbf{x}_{i t}-\mathbf{x}_{i t-1}\right)$ concerning the evolutive features (i.e. the "variational" patterns) of the trajectories measured by means of their absolute variation i.e. $d\left(\mathbf{x}_{i t}, \tilde{\mathbf{x}}_{c t}\right)=\left\|\mathbf{v}_{i t}-\tilde{\mathbf{v}}_{i^{\prime} t}\right\|, t=2, \ldots, T$. Also the percentual variation $\mathbf{v} \mathbf{r}_{i t}=\frac{\left(\mathbf{x}_{i t}-\mathbf{x}_{i t-1}\right)}{\mathbf{x}_{i t-1}} 100$ has been considered i.e. $d\left(\mathbf{x}_{i t}, \tilde{\mathbf{x}}_{c t}\right)=\left\|\mathbf{v} \mathbf{r}_{i t}-\tilde{\mathbf{v r}}{ }_{i^{\prime} t}\right\|, t=2, \ldots, T$.

If in (1) we consider $T=1$ we obtain the Cross Sectional Fuzzy C-Medoids Clustering model with Contiguity constraints (CS-FCMd-C).

In Fig. 1 an example of Cross Sectional and Longitudinal clustering is shown.

\subsection{Some Remarks: Cluster Validity and Contiguity Correlation}

The parameters to be fixed in model (1) are the number of clusters $C$ and the spatial parameter $\beta$.

A widely used cluster validity criterion for selecting $C$ is the fuzzy extension of the Silhouette criterion (Campello and Hruschka 2006).

The Fuzzy Silhouette makes explicit use of the fuzzy partition matrix $\mathbf{U}=\left\{u_{i k}: i=1, \ldots, I ; c=1, \ldots, C\right\}$. It considers the information on the membership degrees contained in the fuzzy partition matrix $\mathbf{U}$ by stressing importance of units concentrated in the vicinity of the cluster prototypes (high membership) while reducing importance of units lying in overlapping areas (small membership). The Fuzzy Silhouette (FS) is defined as follows: 

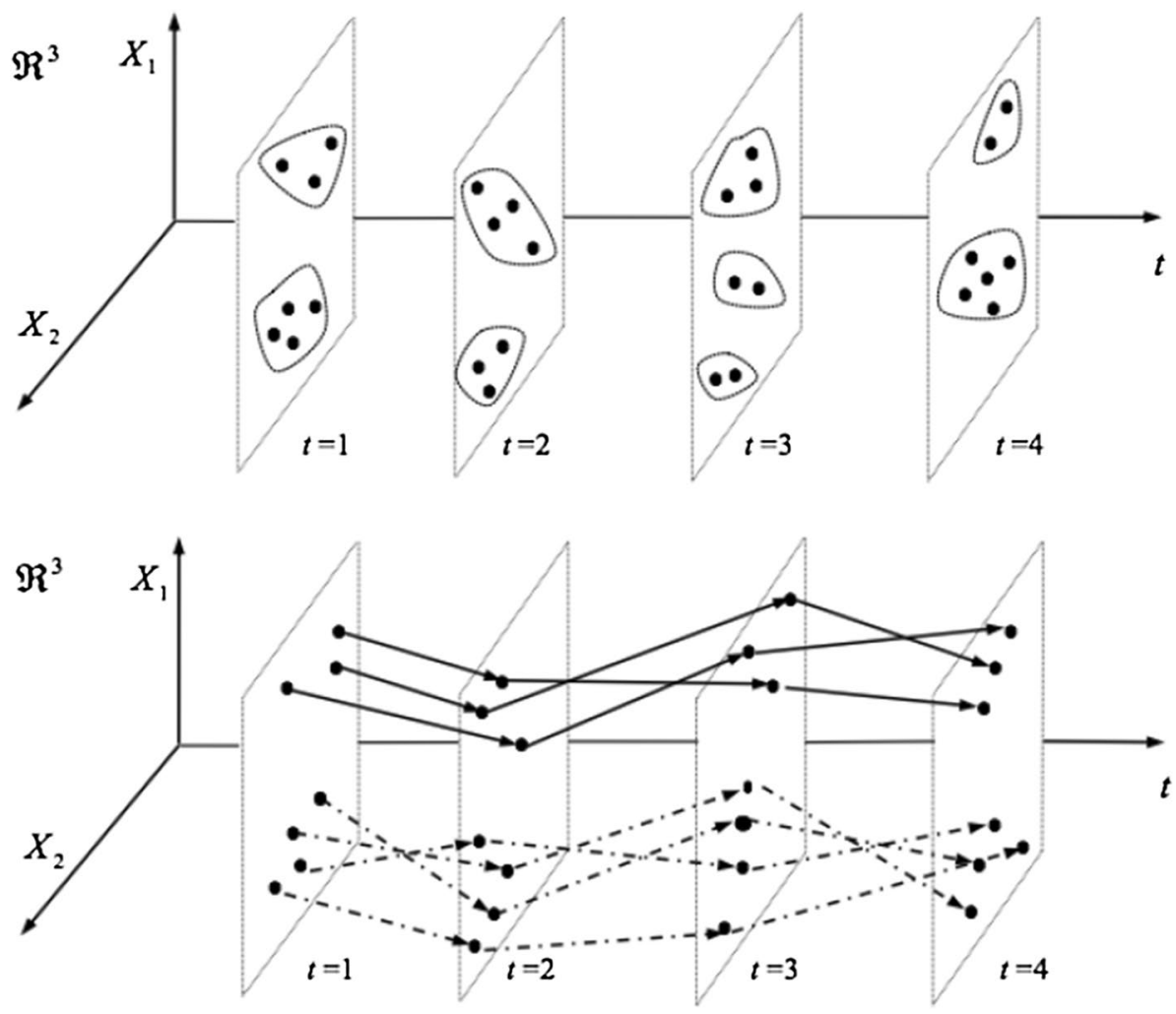

Fig. 1 Example of cross sectional and longitudinal clustering $(I=7, J=2, T=4)$ (D'Urso 2005)

$$
F S=\frac{\sum_{i=1}^{I}\left(u_{i c}-u_{i c^{\prime}}\right)^{\gamma} S_{i}}{\sum_{i=1}^{I}\left(u_{i c}-u_{i c^{\prime}}\right)^{\gamma}} \quad S_{i}=\frac{\left(b_{i}-a_{i}\right)}{\max \left\{b_{i}, a_{i}\right\}}
$$

where $a_{i}$ is the average distance between the $i$-th unit and the units belonging to the cluster $p(p=1, \ldots, C)$ with which $i$ is associated with the highest membership degree; $b_{i}$ is the minimum (over clusters) average distance of the $i$-th unit to all units belonging to the cluster $q$ with $q \neq p, u_{i k}$ and $u_{i k^{\prime}}$ are the first and second largest elements of the $i$-th row of the fuzzy partition matrix, respectively, and $\gamma \geq 0$ is a weighting coefficient. The effect of varying this parameter on the weighting terms in (4) is investigated in Campello and Hruschka (2006).

The selection of the optimal value of $\beta$ is a complex issue. A possible way to solve it is represented by the following heuristic procedure, assuming $C$ and $m$ have already been chosen. For every specified value of $\beta$, the obtained clusters are constructed in such a way that the within cluster dispersion is minimized. However, it would be also desirable that each and every clusters are characterized by the maximal within cluster spatial autocorrelation. To this purpose, for fixed values of $C$ and $m$, it is advisable to run the clustering algorithm for increasing values of $\beta$ (e.g. from 0 to $\beta_{\max }$, with $\beta_{\max }>0$ chosen in advance and with increasing steps equal to $\beta_{\text {inc }}$ ) and to choose the optimal value of $\beta$ in such a way 
that the within cluster spatial autocorrelation is maximized. The measure of contiguity correlation introduced to assess the post-cluster correlation between units, the Fuzzy Moran (FM)'s index, is a multivariate fuzzy generalisation of the Moran's index (Gittleman and Kot 1990). The idea of the FM index is to compute the correlation between classified units in which both the matrix of membership degrees and the contiguity matrix are considered (Coppi et al. 2010). The contiguity correlation measure $\rho_{c}$ for the $c$-th cluster is:

$$
\rho_{c}=\frac{\operatorname{tr}\left(\mathbf{Q} \mathbf{X}_{\text {comp }}\right)^{\prime} \mathbf{U}_{\mathbf{c}}{ }^{0.5} \mathbf{P} \mathbf{U}_{\mathbf{c}}^{0.5}\left(\mathbf{Q} \mathbf{X}_{\text {comp }}\right)}{\operatorname{tr}\left(\mathbf{Q} \mathbf{X}_{\text {comp }}\right)^{\prime} \mathbf{U}_{\mathbf{c}}^{0.5} \mathbf{P}^{\prime} \mathbf{P} \mathbf{U}_{\mathbf{c}}^{0.5}\left(\mathbf{Q} \mathbf{X}_{\text {comp }}\right)}
$$

where $\mathbf{U}_{c}$ is the square diagonal matrix of order $I$ of the membership degrees of cluster $c$; $\mathbf{X}_{\text {comp }}$ is the centred compromise matrix (mean of the $\mathbf{T}$ data matrices $\mathbf{X}_{t}$ ); $\mathbf{P}$ is the contiguity matrix. The matrix $\mathbf{Q}$, in which $\mathbf{Q}=\mathbf{I}_{I}-\frac{\mathbf{1}_{I} \mathbf{1}_{I}^{\prime}}{I}$ is the centering operator, where $\mathbf{I}_{I}$ is an identity matrix of order $I$ and $\mathbf{1}_{I}$ is a column-vector of order $I$ with unit elements.

In order to determine an overall contiguity correlation measure for the obtained partition we can compute the weighted mean of the measures in (5) with weights $s_{c}$ equal to the normalized sum over the $I$ spatial units of the membership degrees in the $C$ clusters:

$$
\rho_{\text {overall }}=\frac{\sum_{c=1}^{C} \rho_{c} s_{c}}{\sum_{c=1}^{C} s_{c}}=\frac{\sum_{c=1}^{C} \rho_{c} s_{c}}{I} .
$$

The contiguity correlation index ranges between -1 and 1 .

\section{Application}

The Fuzzy clustering model with the two dissimilarity measures introduced in Sect. 3 has been applied to the time array represented by 2 times (2013 and 2016), 3 variables (Basic, Efficiency, Innovation) and 256 units (EU NUTS). The interpretation of the clusters has considered all the 11 Pillars composing the RCI. A number of clusters from 3 to 10 has been considered and the number of clusters has been selected on the basis of the validity criteria illustrated in Sect. 3. The parameter $\beta$ has been selected according to the correlation coefficient introduced in Sect. 3. The original 279 EU NUTS have been reduced to 256 by excluding the France NUTS Guadeloupe, Martinique, Guyane, Réunion, Mayotte and Malta (due to missing data) and taking into account the RCI 2016 time comparisons indications to harmonize the 2013 and 2016 NUTS classification.

The application of the models is described in Table 10. The Fuzzy C-Medoids model has been applied without contiguity constraints in the cross-sectional form at the times 2016 (Sect. 4.2) and 2013 (Sect. 4.3) and in the longitudinal form either binding the 2013 and 2016 indicators (Sect. 4.5) or computing the absolute and relative variations between 2016 and 2013 (Sect. 4.4). The Fuzzy C-Medoids model has been applied with contiguity constraints in the cross-sectional form at time 2016 using a geographic contiguity (Sect 4.6) and a level of GDP contiguity (Sect. 4.7). 
Table 10 Models and applications (sections in parenthesis)

\begin{tabular}{lll}
\hline 2013 & 2016 & $2013-2016$ \\
\hline CS-FCMd (4.2) & CS-FCMd (4.3) & L-FCMd (4.5) \\
& CS-FCMd-C (geographic) (4.6) & L-FCMd absolute variation (4.4) \\
& CS-FCMd-C (GDP) (4.7) & L-FCMd relative variation (4.4) \\
\hline
\end{tabular}

\subsection{Cross Sectional Fuzzy C-Medoids Clustering (CS-FCMd) RCI 2016}

A first clustering has been developed on the basis of the univariate RCI 2016 index (Fig. 2). The EU establishes 8 classes of RCI 2016 and associates each class to a color in the maps published. The classes are: $(<-1.00),(-1.00 ;-0.50)$, $(-0.50 ;-0.20),(-0.20 ; 0.00),(0.00 ; 0.20),(0.20 ; 0.50),(0.50 ; 1.00),(>1.00)$ and the classes sizes are (1 2345678$)(294330202562416)$.

The partition in 8 clusters obtained applying the CS-FCMd clustering model is a good partition with respect to the cluster validity index. The obtained centroids for the Fuzzy C-Medoids with 8 clusters are $(-1.32 ;-0.92 ;-0.59 ;-0.27 ; 0.05 ; 0.35 ; 0.630 .97)$ and the cluster sizes are (21 176326233838 30). The medoids of the Fuzzy C-Medoids show the prevalence of low values of RCI 2016.

\subsection{Cross Sectional Fuzzy C-Medoids Clustering (CS-FCMd) RCI 2016 Subindexes}

The Sammon projection (Sammon 1969) $)^{2}$ of the EU regions (Fig. 3) shows that the regions with high positive values of the three subindexes are in the left quadrants (UK00, UKJ1, NL31, SE11 at the most left); then moving to right there are the regions with positive values of the three subindexes; then the regions with negative values of the three subindexes and then the regions with high negative values of the three subindexes (RO22, EL51, EL64, BG31 at the most right). They are the four best and the four worst regions with respect to the value of the RCI. All the Italian regions have a negative value of the RCI (Table 7). It has to be noticed that all the Italian NUTS have negative values of the three subindexes with the exception of Lombardia in the Efficiency subindex.

The best partition is the 6 clusters partition according to the $F S$ index (Sect. 3.2). The clusters are very high negative cluster 2 (medoid EL53), high negative cluster 1 (medoid SK04) and medium negative cluster 3 (medoid ITI3); low positive cluster 4 (medoid FR43), medium positive cluster 5 (medoid SE33) and high positive cluster 6 (medoid $\mathrm{BE} 21$ ). In the high negative cluster the worst subindex is the Basic; in the high positive cluster the best subindex is Innovation (Table 11). The intervals for very high, high, medium and low negative and positive - according to the RCI thresholds (Sect. 4.1) are$(<-1.0) ;(-1.0 ;-0.5) ;(-0.5 ;-0.2) ;(-0.2 ;-0.0) ;(0.0 ; 0.2) ;(0.2 ; 0.5) ;(0.5 ; 1.0) ;(>1.0)$

(Fig. 2). Three clusters have negative values of the subindexes; three clusters have positive values of the subindexes.

\footnotetext{
${ }^{2}$ The Sammon projection is a projection of points onto a low-dimensional space aimed at minimising the error projection obtained summing up the squared differences (before versus after transformation) in pairwise distances between points
} 


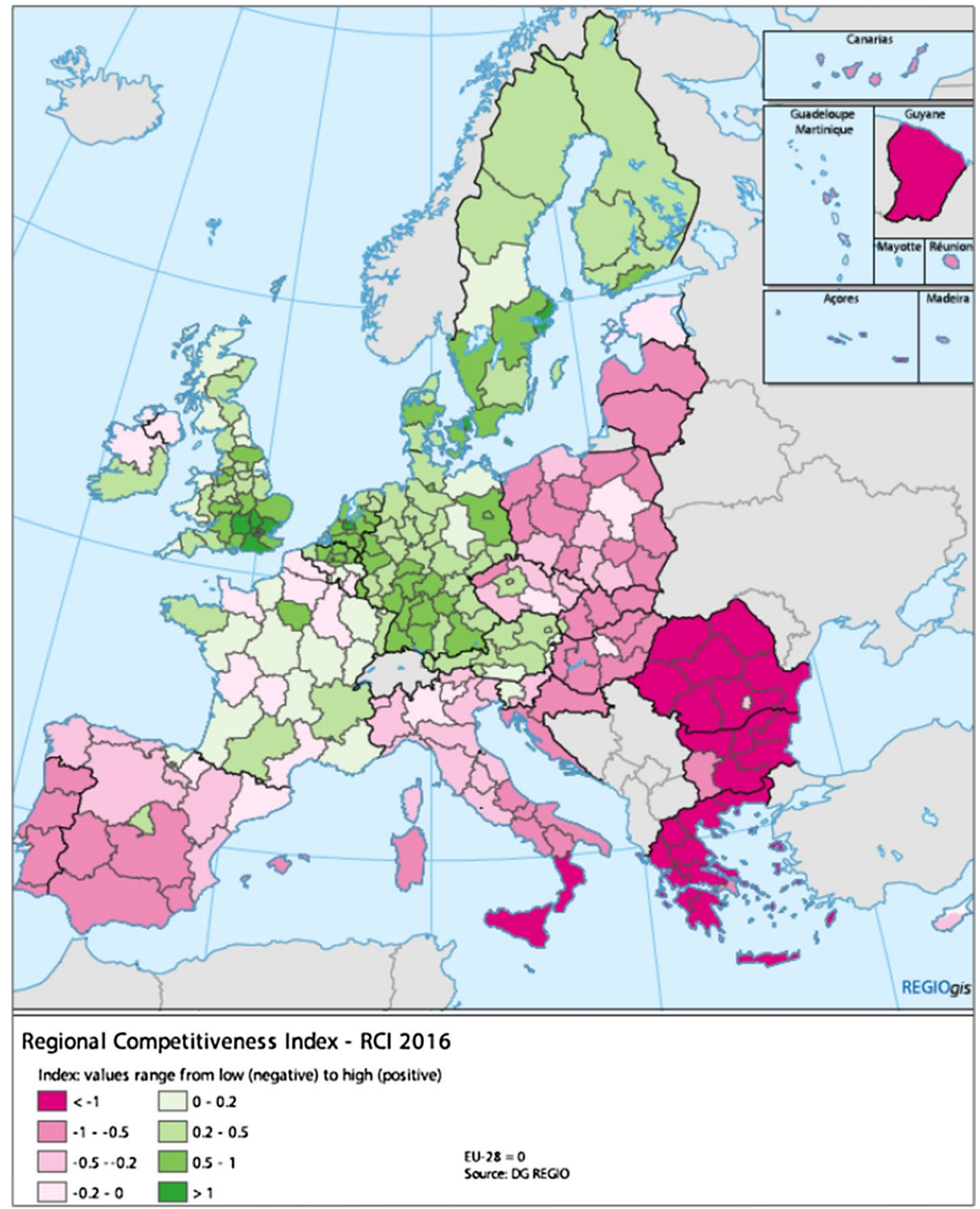

Fig. 2 RCI 2016-Annoni et al. (2017)

Regions Lombardia (ITC4) and Lazio (ITI4) are in cluster 4 (low positive), close in Fig. 3; the regions Campania (ITF3), Puglia (ITF4), Basilicata (ITF5), Calabria (ITF6), Sicilia (ITG1), Sardegna (ITG2) are in cluster 1 (high negative) and the others in cluster 3 (medium negative). The only region with membership smaller than 0.50 is ITH2 that shows 0.493 membership to cluster 2 and 0.462 to cluster 4 (Table 13).

The regions in cluster 1 (Table 12) are mostly regions of Spain (ES), Hungary (HU) and Italy (IT); the regions in cluster 2 mostly regions mostly regions of Bulgaria (BG), Greece (EL) and Romania (RO); the regions in cluster 3 mostly regions of Czech Republic (CZ), 


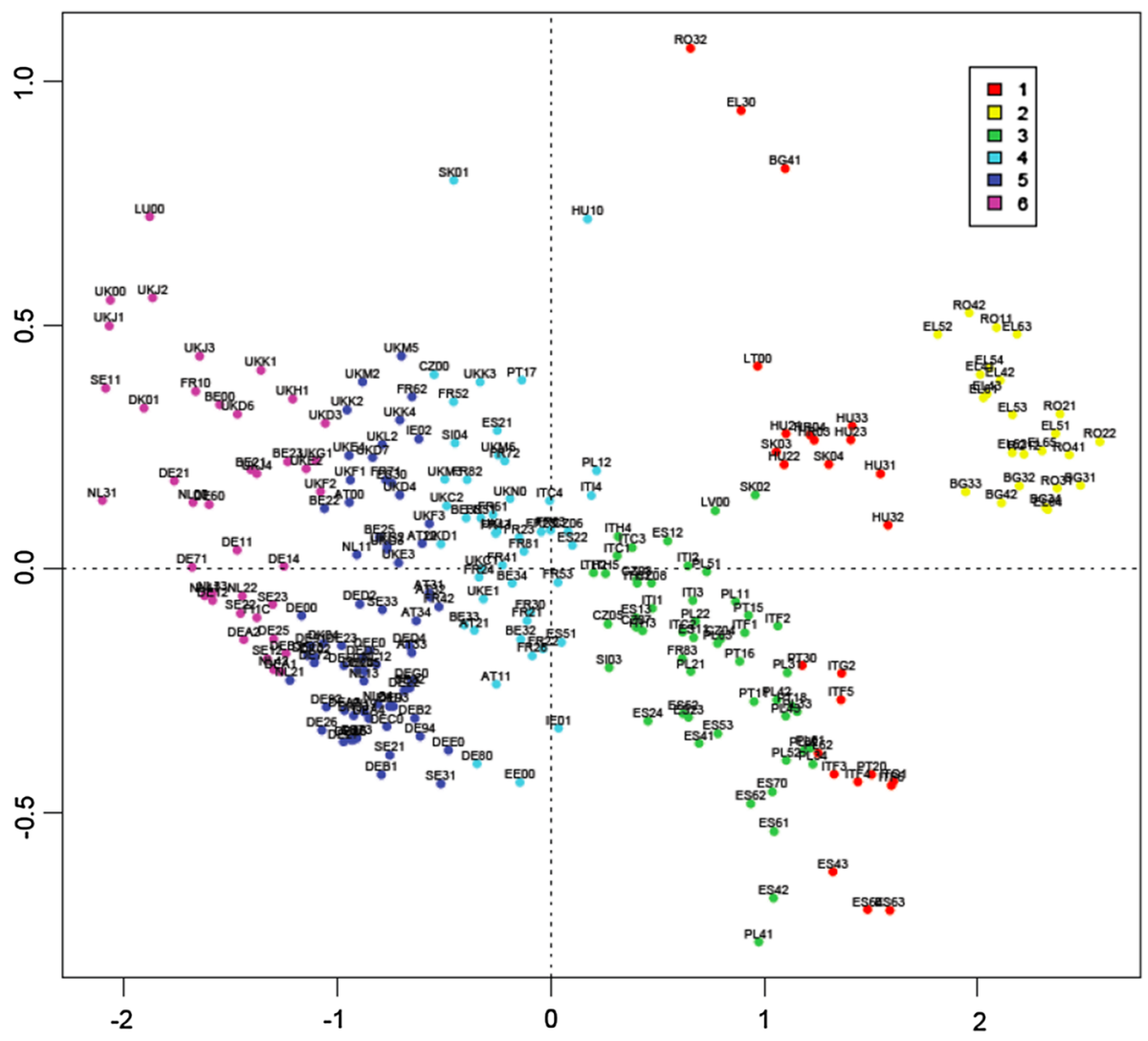

Fig. 3 Sammon projection of the NUTS and clustering

Table 11 Fuzzy C-medoids RCI 2016 clusters

\begin{tabular}{lrrrl}
\hline Medoid & Basic & Efficiency & Innovation & \\
\hline 1 SK04 Východné Slovensko & -0.943 & -0.811 & -0.777 & ITF3, ITF4, ITF5 \\
& & & & ITF6, ITG1, ITG2 \\
2 EL53 Dytiki Makedonia & -1.485 & -1.237 & -1.301 & \\
3 ITI3 Marche & -0.355 & -0.448 & -0.590 & Others \\
4 FR43 Franche-Comté & 0.017 & 0.074 & 0.094 & ITC4, ITI4 \\
5 SE33 Övre Norrland & 0.434 & 0.278 & 0.396 & \\
6 BE21 Antwerpen & 0.526 & 0.722 & 0.902 & \\
\hline
\end{tabular}

Spain (ES), Italy (IT) and Poland (PL); the regions in cluster 4 mostly regions of Belgium (BE), Spain (ES), France (FR) and United Kingdom (UK); the regions in cluster 5 mostly regions of Austria (AT), Germany (DE), Netherlands (NL) and United Kingdom (UK) and regions in cluster 6 mostly regions of Germany (DE), Netherland (NL), Sweden (SE) and United Kingdom (UK). 
Table 12 Fuzzy C-medoids RCI 2016 cluster composition

\begin{tabular}{|c|c|c|c|c|c|c|c|c|c|c|c|c|c|}
\hline & AT & $\mathrm{BE}$ & BG & $\mathrm{CZ}$ & $\mathrm{DE} \quad \mathrm{I}$ & DK E & EE EL & ES & FI & FR & HR & $\mathrm{HU}$ & IE \\
\hline Cluster 1 & & & 1 & & & & 1 & 3 & & & 2 & 6 & \\
\hline Cluster 2 & & & 5 & & & & 12 & & & & & & \\
\hline Cluster 3 & & & & 5 & & & & 12 & & 1 & & & \\
\hline Cluster 4 & & 4 & & 2 & 1 & 1 & 1 & 3 & & 17 & & 1 & 1 \\
\hline Cluster 5 & 2 & 2 & & & 26 & 4 & & 1 & 3 & 3 & & & 1 \\
\hline \multirow[t]{2}{*}{ Cluster 6} & 6 & 3 & & & 10 & 1 & & & 1 & 1 & & & \\
\hline & 8 & 9 & 6 & 7 & 37 & 1 & 13 & 19 & 4 & 22 & 2 & 7 & 2 \\
\hline Gini heterogeneity & 0.45 & 0.77 & 0.33 & 0.49 & 0.52 & $0.38 \quad 0$. & $\begin{array}{ll}0.00 & 0.17\end{array}$ & 0.66 & 0.45 & 0.46 & 0.00 & 0.29 & 0.60 \\
\hline \multirow[t]{2}{*}{$\%$ in clusters $4-6$} & 1.00 & 1.00 & 0.00 & 0.29 & 1.00 & 1.001. & $1.00 \quad 0.00$ & 0.21 & 1.00 & 0.95 & 0.00 & 0.14 & 1.00 \\
\hline & IT & LT & LU & LV & NL & PL & PT & RO & SE & SI & SK & UK & \\
\hline Cluster 1 & 6 & 1 & & & & 1 & 2 & 1 & & & 2 & & 26 \\
\hline Cluster 2 & & & & & & & & 7 & & & & & 24 \\
\hline Cluster 3 & 13 & & & 1 & & 14 & 4 & & & 1 & 1 & & 52 \\
\hline Cluster 4 & 2 & & & & & 1 & 1 & & & 1 & 1 & 9 & 47 \\
\hline Cluster 5 & & & & & 5 & & & & 4 & & & 13 & 68 \\
\hline \multirow[t]{2}{*}{ Cluster 6} & & & 1 & & 6 & & & & 4 & & & 12 & 39 \\
\hline & 21 & 1 & 1 & 1 & 11 & 16 & 7 & 8 & 8 & 2 & 4 & 34 & 256 \\
\hline Gini heterogeneity & 0.63 & 0.00 & 0.00 & 0.00 & $\begin{array}{ll}0 & 0.60\end{array}$ & $\begin{array}{ll}0 & 0.27\end{array}$ & $7 \quad 0.69$ & 0.26 & 0.60 & 0.60 & 0.75 & 0.79 & \\
\hline$\%$ in clusters $4-6$ & 0.10 & 0.00 & 1.00 & 0.00 & $\begin{array}{ll}0 & 1.00\end{array}$ & $\begin{array}{ll}0 & 0.06\end{array}$ & $\begin{array}{ll}6 & 0.14\end{array}$ & 0.00 & 1.00 & 0.50 & 0.25 & 1.00 & \\
\hline
\end{tabular}

For each country the normalized Gini heterogeneity index of dispersion (range in $0-1,0$ minimum heterogeneity) of the NUTS among the clusters is presented in Table 12 together with the percentage of NUTS in the clusters with positive values of the subindexes to join the information about the dispersion among clusters with the membership to good clusters. The indexes show that NUTS in Western Europe countries (Austria, Belgium, France, Germany, Ireland, Luxembourg, The Netherlands, United Kingdom) and in Northern Europe countries (Denmark, Estonia, Finland, Latvia, Lituania, Sweden) are sparsed among the good clusters.

The membership to all the clusters for the Italian regions is presented in Table 13; the highest membership $(U)$ and related cluster for the NUTS is presented in Table 14.

As a general comment the regions of the NUTS1 level NORD-OVEST and NORDEST perform better than the others.

Lombardia (ITC4) is the best performing among the 20 Italian regions with a standardized score equal to -0.047 , which corresponds to a rank of 158 out 263 (was 128th in 2013). As in 2013, the only positive score is reached in the Efficiency subindex $(+0,045)$ even if it has deteriorated from +0.114 registered three years before. With regard to the pillars the best position is reached in Health (rank 32th), followed by the Business Sophistication (rank 59th) and Infrastructure (rank 69th). It is worth noting that Lombardia is the most prosperous region in Italy, with a GDP per capita equal to 33,5 thousand euro about $31 \%$ higher than the European average. It is also the leading region in the Italian economy as measured by total production, export and employment representing over one fifth of the whole national value. Despite the strengths and 
Table 13 Fuzzy C-Medoids RCI 2016 memberhip

\begin{tabular}{|c|c|c|c|c|c|c|c|}
\hline Year & NUTS & Cluster 1 & Cluster 2 & Cluster 3 & Cluster 4 & Cluster 5 & Cluster 6 \\
\hline 2016 & ITC1 & 0.017 & 0.001 & 0.809 & 0.159 & 0.012 & 0.002 \\
\hline 2016 & ITC2 & 0.000 & 0.000 & 1.000 & 0.000 & 0.000 & 0.000 \\
\hline 2016 & ITC3 & 0.013 & 0.001 & 0.919 & 0.060 & 0.005 & 0.001 \\
\hline 2016 & ITC4 & 0.002 & 0.000 & 0.025 & 0.958 & 0.013 & 0.002 \\
\hline 2016 & ITF1 & 0.053 & 0.001 & 0.943 & 0.002 & 0.001 & 0.000 \\
\hline 2016 & ITF2 & 0.375 & 0.008 & 0.611 & 0.005 & 0.001 & 0.000 \\
\hline 2016 & ITF3 & 0.732 & 0.061 & 0.191 & 0.011 & 0.004 & 0.001 \\
\hline 2016 & ITF4 & 0.670 & 0.117 & 0.191 & 0.014 & 0.005 & 0.002 \\
\hline 2016 & ITF5 & 0.771 & 0.048 & 0.172 & 0.006 & 0.002 & 0.001 \\
\hline 2016 & ITF6 & 0.632 & 0.211 & 0.139 & 0.012 & 0.005 & 0.002 \\
\hline 2016 & ITG1 & 0.623 & 0.224 & 0.134 & 0.012 & 0.005 & 0.002 \\
\hline 2016 & ITG2 & 0.846 & 0.042 & 0.104 & 0.005 & 0.002 & 0.001 \\
\hline 2016 & ITH1 & 0.020 & 0.002 & 0.894 & 0.072 & 0.010 & 0.002 \\
\hline 2016 & ITH2 & 0.015 & 0.002 & 0.493 & 0.462 & 0.025 & 0.004 \\
\hline 2016 & ITH3 & 0.005 & 0.000 & 0.977 & 0.016 & 0.002 & 0.000 \\
\hline 2016 & ITH4 & 0.018 & 0.001 & 0.804 & 0.163 & 0.012 & 0.002 \\
\hline 2016 & ITH5 & 0.015 & 0.001 & 0.695 & 0.269 & 0.017 & 0.003 \\
\hline 2016 & ITI1 & 0.003 & 0.000 & 0.989 & 0.006 & 0.001 & 0.000 \\
\hline 2016 & ITI2 & 0.000 & 0.000 & 1.000 & 0.000 & 0.000 & 0.000 \\
\hline 2016 & ITI3 & 0.000 & 0.000 & 1.000 & 0.000 & 0.000 & 0.000 \\
\hline 2016 & ITI4 & 0.020 & 0.002 & 0.325 & 0.619 & 0.030 & 0.005 \\
\hline
\end{tabular}

Bold values indicate highest membership

resiliency of its economy during the global recession, Lombardia faces critical challenges: improving the overall educational attainment levels and upgrading the skills of the regional population by aligning higher education provision with the needs and opportunities of the region and its SMEs (OECD 2011). The region is undergoing an industrial shift towards services and knowledge-intensive activities and the manufacturing firms will need to focus on differentiation and a more intense use of knowledge. The success of this emerging manufacturing model relies on the access to skilled and qualified labour: in 2016 the percentage of population aged 25-64 with a tertiary educational attainment is $19.3 \%$ (215th the rank) compared to $74.8 \%$ of Inner London, the best competitive region in Europe (Eurostat database).

Lazio (ITI4) is indeed the administrative centre of Italy and therefore the business sector is less important than in most of the other central and northern Italian regions. Lazio is the Italian region that is more oriented towards services: about $85 \%$ of its value added (at current market prices) is related to services, of which $33 \%$ to financial and insurance, real estate, professional, scientific and technical activities. As regard to Basic subindex, Lazio in recent years has taken effective actions in order to enhance the quality of their institutions (247th its rank) by simplifying the life of citizens and enterprises: a single SUAP (One stop shop for Production Activities) with a single information system, same forms to be used everywhere in the region, a homogeneous offer with services for all the Lazio towns. Furthermore, Lazio has implemented a plurality of interventions supporting the 


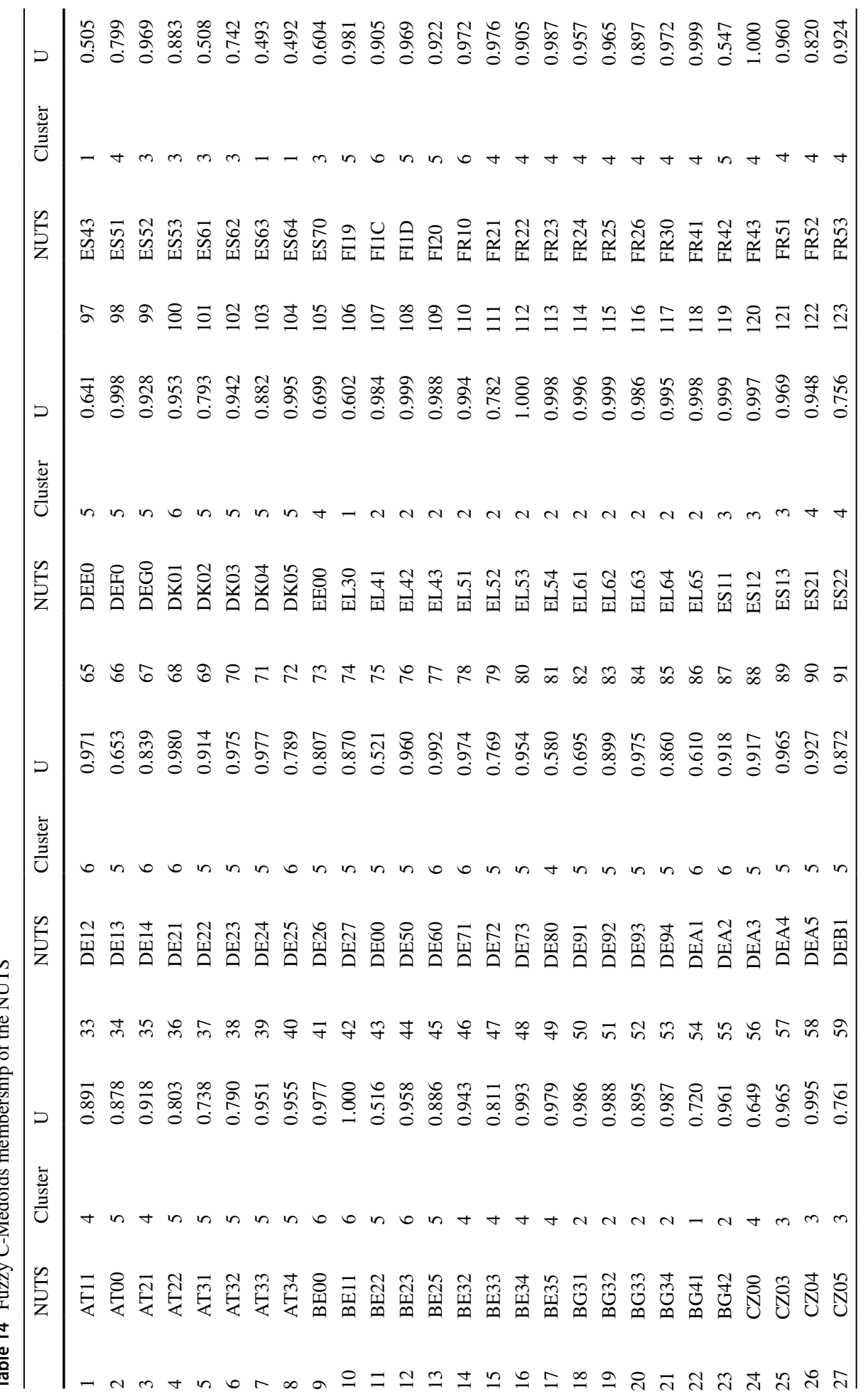




\begin{tabular}{|c|c|c|c|}
\hline 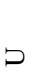 & 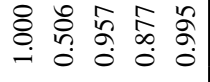 & $\triangleright$ & 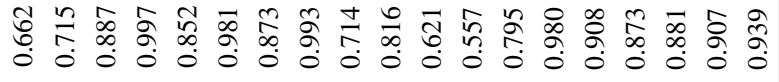 \\
\hline 岕 & $\nabla n+\backsim \nabla$ & 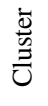 & +0 in 0 in +0 in $n$ in 6 in 6 in in 6006 \\
\hline 管 & 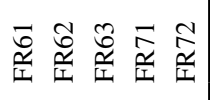 & $\stackrel{n}{5}$ & 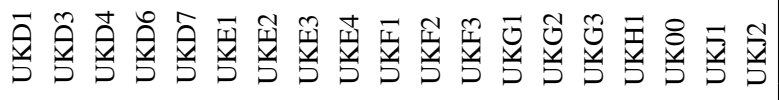 \\
\hline & 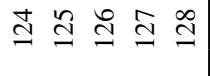 & & 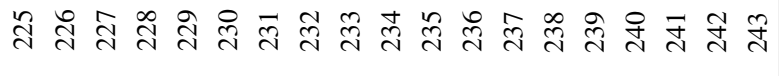 \\
\hline$\triangleright$ & 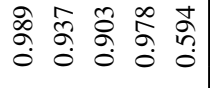 & $\supset$ & 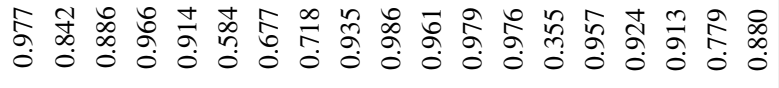 \\
\hline$\frac{\bar{\Xi}}{\stackrel{\Xi}{\Xi}}$ & $n n n m m$ & 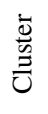 & 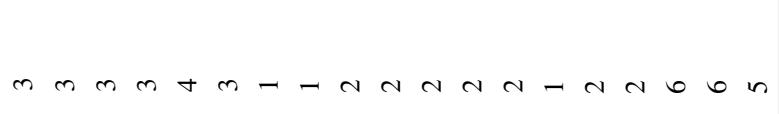 \\
\hline 先 & 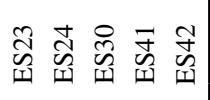 & $\stackrel{n}{5}$ & 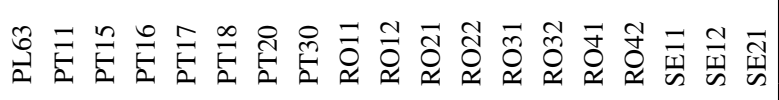 \\
\hline & 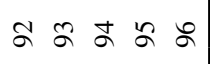 & & 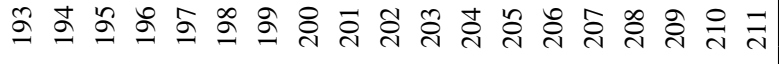 \\
\hline$\supset$ & 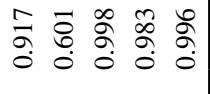 & $\triangleright$ & 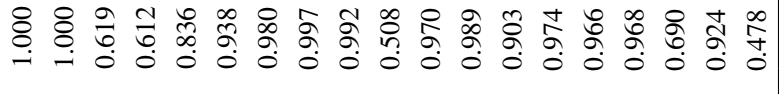 \\
\hline 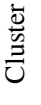 & $n$ in $n$ in $n$ & 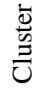 & 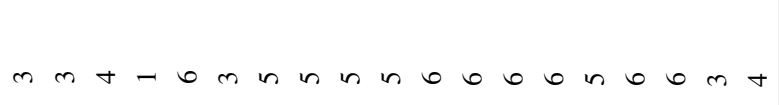 \\
\hline$\overbrace{\xi}^{2}$ & 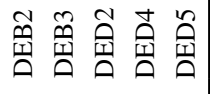 & $\stackrel{n}{\Leftrightarrow}$ & 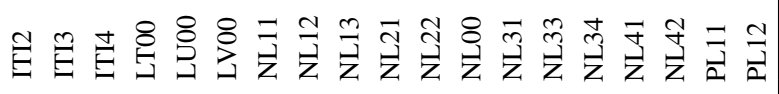 \\
\hline & 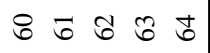 & & 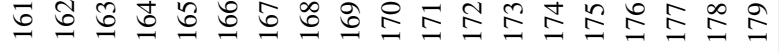 \\
\hline$\triangleright$ & 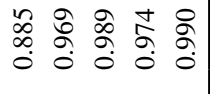 & $\supset$ & 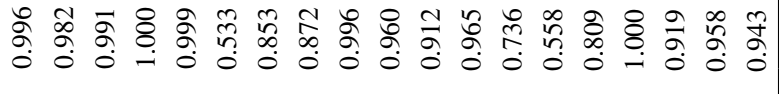 \\
\hline 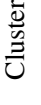 & 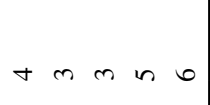 & 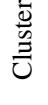 & 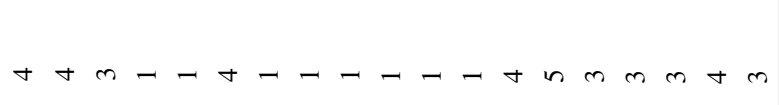 \\
\hline 笘 & 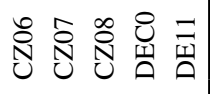 & $\stackrel{n}{\overbrace{2}}$ & 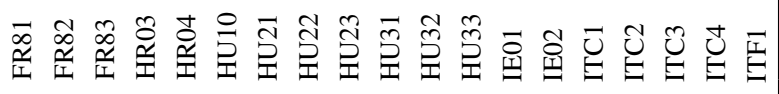 \\
\hline & & & 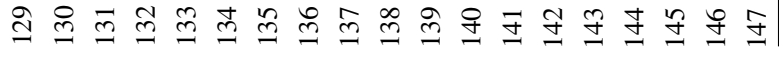 \\
\hline
\end{tabular}




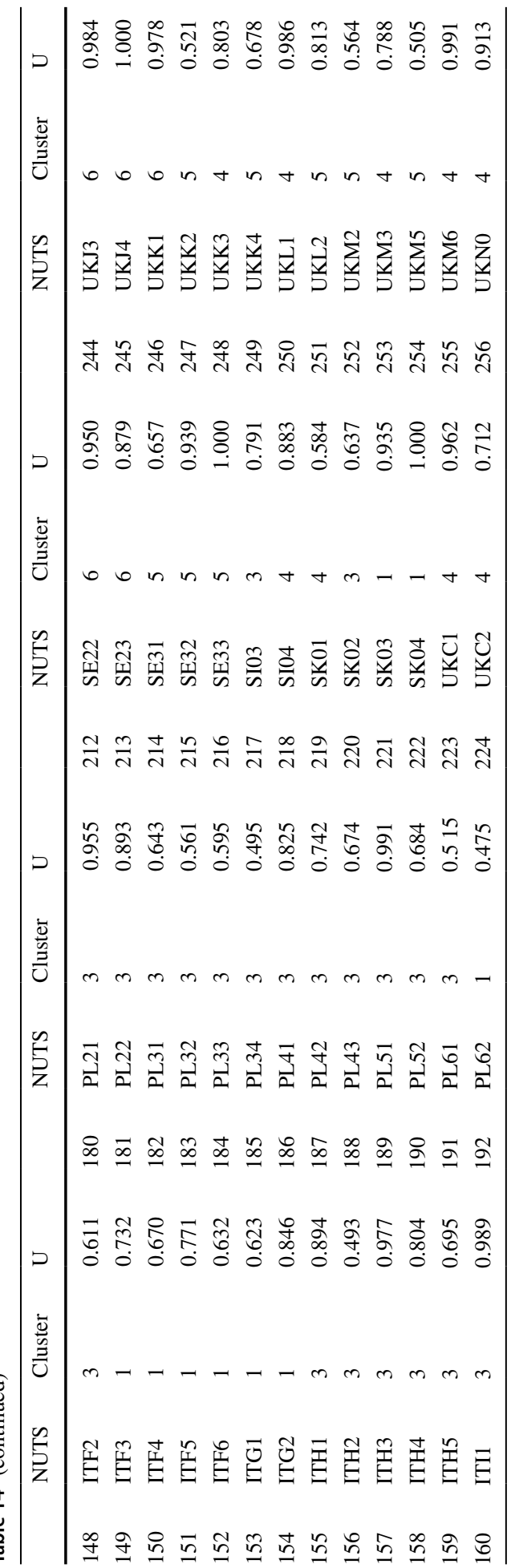


Table 15 Fuzzy C-Medoids RCI 2013 clusters

\begin{tabular}{llcll}
\hline Medoid & Basic & Efficiency & Innovation & \\
\hline BG33 Severoiztochen & -1.360 & -1.232 & -1.332 & \\
ES13 Cantabria & -0.141 & -0.255 & -0.439 & Others \\
PL42 Zachodniopomorskie & -0.434 & -0.821 & -0.885 & ITF1, ITF2, ITF3 \\
& & & & ITF4, ITF5, ITF6 \\
& & & & ITG1, ITG2 \\
DE11 Stuttgart & 0.693 & 0.841 & 0.975 & \\
FR23 Haute-Normandie & 0.002 & -0.226 & 0.077 & ITC4, ITI4 \\
DE23 Oberpfalz & 0.485 & 0.414 & 0.426 & \\
\hline
\end{tabular}

reconversion of the productive system based on strengthening the networks and consortia of enterprises and supporting the propensity to innovate and the technological transfer. With respect to Innovation subindex, Lazio with a score of -0.08 (143th) has performed even better than Lombardia $(-0.12,148$ th $)$. This result depends from the fact that it is a unique research and knowledge area in Italy and, indeed, presents a high concentration of public and private research institutes, technological poles and University institutions on an international level: there are more than 200 research laboratories, more than 40 public research institutes, 6 public universities, and 4 Centres of Excellence, such as the National Research Council (with more than 50 departments), ENEA (Institute for Energy), the National Institute for Nuclear Physics and the National Institute of Health. In total, the public sector employs $72 \%$ of total R\&D staff. Human resources involved in research and development amounted to 150,700 about $6.5 \%$ of the total active population, higher than the Italian (6.1\%) and European (5.8\%) average. But patenting activity is however weak: only 24 patents per million inhabitants where generated in 2012, significantly less than the Italian (60) and European (112) average. Finally, with respect to the diffusion of ICTs, in 2016, Lazio has registered a good percentage of households with internet broadband access (81\%), above the Italian average (77\%) and not so far from the one for Europe (88\%).

It important to underline that the decline of the scores for Abruzzo in 2016 is influenced by the devastating earthquake which occurred in 2009. Abruzzo (ITF1) is the one of the most industrialised region in Southern Italy (34.2\% the share of value added from industry): the industrialisation rate is above the Italian average (73 enterprises per 1000 residents vs. a national average of 72).

\subsection{Cross Sectional Fuzzy C-Medoids Clustering (CS-FCMd) RCI 2013 Subindexes}

In 2013 the Italian regions except Lombardia (ITC4) have e negative value of the RCI. Lombardia (ITC4), Emilia Romagna (ITH5), Lazio (ITI4) and Puglia (ITF4), Calabria (ITF6), Sicilia (ITG1) are the three best and three worst italian regions with respect to the value of the RCI.

The best partition is the 6 clusters partition according to the $F S$ index (Sect. 3.2). The clusters are very high negative cluster 1 (medoid BG33), high negative cluster 3 (medoid PL42) and medium negative cluster 2 (medoid ES13); low positive cluster 5 (medoid FR23), medium positive cluster 6 (medoid DE23) and high positive cluster 4 (medoid DE11). In the low negative cluster all the subindexes have a value smaller than -1.00 and 
Table 16 Fuzzy C-Medoids RCI 2013 memberhip

\begin{tabular}{|c|c|c|c|c|c|c|c|}
\hline Year & NUTS & Cluster 1 & Cluster 2 & Cluster 3 & Cluster 4 & Cluster 5 & Cluster 6 \\
\hline 2013 & ITC1 & 0.000 & 0.927 & 0.002 & 0.000 & 0.069 & 0.001 \\
\hline 2013 & ITC2 & 0.001 & 0.886 & 0.097 & 0.000 & 0.013 & 0.001 \\
\hline 2013 & ITC3 & 0.000 & 0.782 & 0.004 & 0.000 & 0.210 & 0.003 \\
\hline 2013 & ITC4 & 0.000 & 0.041 & 0.001 & 0.001 & 0.952 & 0.006 \\
\hline 2013 & ITF1 & 0.004 & 0.323 & 0.657 & 0.000 & 0.014 & 0.002 \\
\hline 2013 & ITF2 & 0.002 & 0.015 & 0.982 & 0.000 & 0.001 & 0.000 \\
\hline 2013 & ITF3 & 0.008 & 0.030 & 0.955 & 0.000 & 0.006 & 0.001 \\
\hline 2013 & ITF4 & 0.013 & 0.012 & 0.971 & 0.000 & 0.003 & 0.001 \\
\hline 2013 & ITF5 & 0.003 & 0.003 & 0.993 & 0.000 & 0.000 & 0.000 \\
\hline 2013 & ITF6 & 0.019 & 0.010 & 0.968 & 0.000 & 0.002 & 0.000 \\
\hline 2013 & ITG1 & 0.062 & 0.031 & 0.895 & 0.001 & 0.008 & 0.002 \\
\hline 2013 & ITG2 & 0.006 & 0.013 & 0.978 & 0.000 & 0.002 & 0.000 \\
\hline 2013 & ITH1 & 0.000 & 0.988 & 0.007 & 0.000 & 0.004 & 0.000 \\
\hline 2013 & ITH2 & 0.000 & 0.930 & 0.003 & 0.000 & 0.064 & 0.002 \\
\hline 2013 & ITH3 & 0.000 & 0.997 & 0.001 & 0.000 & 0.002 & 0.000 \\
\hline 2013 & ITH4 & 0.000 & 0.938 & 0.004 & 0.000 & 0.056 & 0.001 \\
\hline 2013 & ITH5 & 0.001 & 0.648 & 0.010 & 0.002 & 0.329 & 0.011 \\
\hline 2013 & ITI1 & 0.000 & 0.983 & 0.002 & 0.000 & 0.014 & 0.000 \\
\hline 2013 & ITI2 & 0.000 & 0.986 & 0.008 & 0.000 & 0.006 & 0.000 \\
\hline 2013 & ITI3 & 0.001 & 0.920 & 0.065 & 0.000 & 0.013 & 0.001 \\
\hline 2013 & ITI4 & 0.000 & 0.123 & 0.004 & 0.001 & 0.866 & 0.006 \\
\hline
\end{tabular}

Bold values indicate highest membership

the worst subindexes are Basic and Innovation; in the high positive cluster all the subindexes have a value between 0,50 and 1,00 and the best subindex is Innovation (Table 15).

Regions Lombardia (ITC4) and Lazio (ITI4) are in cluster 5 (low positive); the regions Abruzzo (ITF1), Molise (ITF2), Campania (ITF3), Puglia (ITF4), Basilicata (ITF5), Calabria (ITF6), Sicilia (ITG1), Sardegna (ITG2) are in cluster 3 (medium negative) and the others in cluster 2 (low negative). It has to be noticed that all the Italian NUTS have negative values of the three subindexes with the exception of Lombardia (ITC4) and Emilia Romagna (ITH5) in the Efficiency subindex and Lazio (ITI4) in the Innovation subindex.

The membership to all the clusters for Italian regions is presented in Table 16.

All the Italian regions have a value of RCI in 2016 smaller than in 2013 with the exception of Bolzano (ITH1), even if some regions have improved the value of a subindex.

\subsection{Longitudinal Fuzzy C-Medoids Clustering (L-FCMd) RCI Subindexes (Absolute and Percentual Variation 2013-2016)}

The histogram of the absolute variations in the subindexes Basic, Efficiency and Innovation for the 256 NUTS are presented in Fig. 4. The modal class of the absolute variations among the EU RCI classes (Sect. 4.1) is $(0.0 ; 0.2)$ for the Basic subindex; $(-0.20 ; 0.00)$ for the Efficiency and Innovation subindex. Positive variations 2013-2016 are registered 

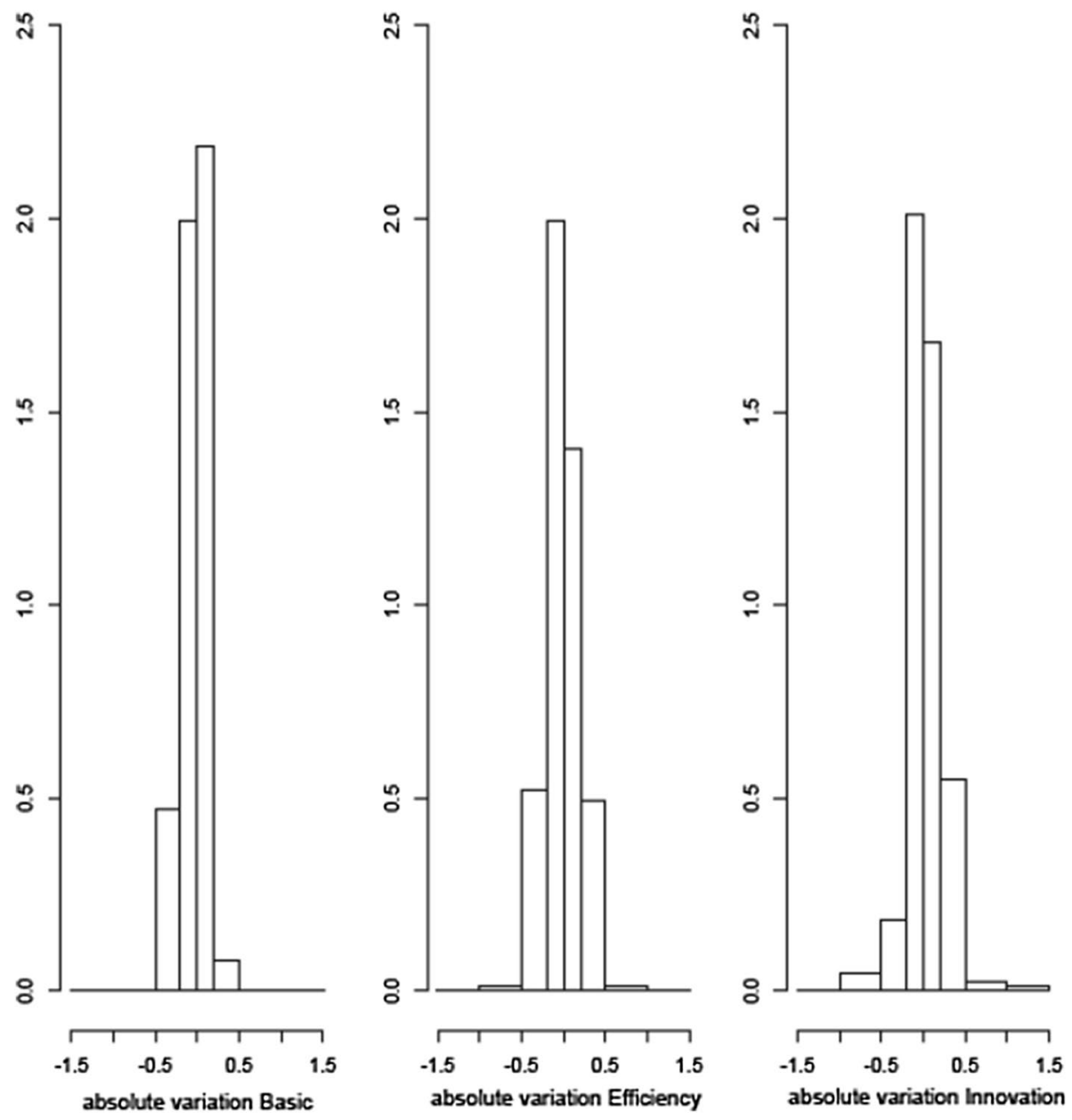

Fig. 4 Subindexes absolute variation 2013-2016

mostly in the Basic subindex. The absolute and percentual variations 2013-2016 for Italy with respect to the 3 subindexes are presented in Table 8 .

The best partition according to the $F S$ index (Sect. 3.2) is the 5 clusters partition. The five clusters have in general small negative values of the absolute variation 2013-2016 for each subindex. Beside two small negative values, cluster 1 medium positive efficiency (medoid FR83) shows a medium positive variation in the subindex Efficiency; cluster 2 low positive innovation (medoid PT17) a small positive variation in the subindex Innovation; cluster 3 medium negative efficiency (medoid ITC3) a medium negative variation in the subindex Efficiency; cluster 4 medium positive innovation (medoid UKH1) a medium positive variation in the subindex Innovation; cluster 5 low positive efficiency (medoid RO21) shows a small positive variation in the subindex Efficiency (Table 17).

Italian regions Lombardia (ITC1), Valle d'Aosta (ITC2), Molise (ITF2), Trento (ITH2), Friuli Venezia Giulia (ITH4), Marche (ITI3) are in cluster 2 low positive innovation. Italian regions Lombardia (ITC4), Basilicata (ITF5), Veneto (ITH3) and Lazio 
Table 17 Fuzzy C-Medoids RCI absolute variation 2013-2016 clusters

\begin{tabular}{lcccl}
\hline Medoid & Basic & Efficiency & Innovation & \\
\hline 1 FR83 Corse & -0.030 & 0.239 & -0.022 & ITH1 \\
2 PT17 Lisboa & -0.014 & -0.069 & 0.068 & ITC1, ITC2, ITF2 \\
& & & & ITH2, ITH4, ITI3 \\
3 ITC3 Liguria & -0.096 & -0.217 & -0.071 & Others \\
4 UKH1 East Anglia & -0.009 & -0.055 & 0.329 & \\
5 RO21 Nord-Est (RO) & -0.020 & 0.005 & -0.003 & ITC4, ITF5, ITH3, ITI4 \\
\hline
\end{tabular}

Table 18 Fuzzy C-Medoids absolute variation membership

\begin{tabular}{|c|c|c|c|c|c|c|}
\hline Year & NUTS & Cluster 1 & Cluster 2 & Cluster 3 & Cluster 4 & Cluster 5 \\
\hline 2013-2016 & ITC1 & 0.004 & 0.450 & 0.326 & 0.005 & 0.215 \\
\hline 2013-2016 & ITC2 & 0.000 & 0.999 & 0.000 & 0.000 & 0.001 \\
\hline 2013-2016 & ITC3 & 0.000 & 0.000 & 1.000 & 0.000 & 0.000 \\
\hline 2013-2016 & ITC4 & 0.006 & 0.290 & 0.105 & 0.003 & 0.595 \\
\hline 2013-2016 & ITF1 & 0.003 & 0.358 & 0.547 & 0.007 & 0.084 \\
\hline 2013-2016 & ITF2 & 0.000 & 0.977 & 0.001 & 0.000 & 0.022 \\
\hline 2013-2016 & ITF3 & 0.016 & 0.073 & 0.808 & 0.008 & 0.094 \\
\hline 2013-2016 & ITF4 & 0.011 & 0.113 & 0.730 & 0.006 & 0.140 \\
\hline 2013-2016 & ITF5 & 0.127 & 0.074 & 0.028 & 0.004 & 0.767 \\
\hline 2013-2016 & ITF6 & 0.018 & 0.095 & 0.768 & 0.011 & 0.108 \\
\hline 2013-2016 & ITG1 & 0.048 & 0.129 & 0.586 & 0.014 & 0.223 \\
\hline 2013-2016 & ITG2 & 0.017 & 0.082 & 0.742 & 0.006 & 0.153 \\
\hline 2013-2016 & ITH1 & 0.987 & 0.002 & 0.000 & 0.000 & 0.010 \\
\hline 2013-2016 & ITH2 & 0.002 & 0.536 & 0.006 & 0.001 & 0.455 \\
\hline 2013-2016 & ITH3 & 0.007 & 0.384 & 0.184 & 0.005 & 0.421 \\
\hline 2013-2016 & ITH4 & 0.001 & 0.877 & 0.017 & 0.001 & 0.104 \\
\hline 2013-2016 & ITH5 & 0.006 & 0.158 & 0.756 & 0.013 & 0.067 \\
\hline 2013-2016 & ITI1 & 0.006 & 0.138 & $\mathbf{0 . 7 5 0}$ & 0.006 & 0.100 \\
\hline 2013-2016 & ITI2 & 0.003 & 0.112 & 0.782 & 0.002 & 0.101 \\
\hline 2013-2016 & ITI3 & 0.002 & 0.730 & 0.023 & 0.002 & 0.243 \\
\hline 2013-2016 & ITI4 & 0.014 & 0.181 & 0.279 & 0.005 & 0.520 \\
\hline
\end{tabular}

Bold values indicate highest membership

(ITI4) are in cluster 5 low positive efficiency. All the other Italian regions are in cluster 3 medium negative efficiency. Two regions have a membership less than 0.50 (Table 18): ITH3 0.421 to cluster 4 and 0.384 to cluster 2; ITC1 0.450 to cluster 2 and 0.326 to cluster 3. The regions in cluster 1 are mostly (first three) regions of France (FR), Poland (PL) and Sweden (SE); the regions in cluster 2 mostly regions of Germany (DE), Spain (ES) and Italy (IT); the regions in cluster 3 mostly regions of Greece (EL), Hungary (HU) and Netherland (NL); the regions in cluster 4 mostly regions of Czech Republic 
Table 19 Fuzzy C-medoids RCI relative variation $2013-2016$ clusters

\begin{tabular}{lrrrr}
\hline Medoid & \multicolumn{1}{c}{ Basic } & Efficiency & Innovation & \\
\hline 1 LV00 Latvija & 0.150 & 0.442 & 0.331 & ITH1 \\
2 CZ07 Strední Morava & 0.407 & 0.233 & 0.418 & \\
3 AT22 Steiermark & 3.889 & 0.423 & 0.887 & \\
4 AT32 Salzburg & 2.731 & 0.257 & 1.150 & \\
5 PL52 Opolskie & -0.006 & -0.110 & -0.057 & Others \\
\hline
\end{tabular}

(CZ), Germany (DE) and United Kingdom (UK); the regions in cluster 5 mostly regions of Germany (DE).

The L-FCMd model has been applied also to the percentual variations 2013-2016 (Table 19).

Considering Italian regions in the Fuzzy C-Medoids Bolzano (ITH1) is alone in cluster 1 medium positive efficiency while all the others are in cluster 5 characterized by small negative percentual variations. The interpretation of the obtained clusters is based also on Table 8 .

Bolzano (ITH1) turns out to be the first region of Italy with the highest GDP per capita: 39,400 EUR in PPs 2014, about $150 \%$ of the Italian and the EU average values. The level of relative wealth has increased with respect to 2008 (37,200), even though the global financial crisis affected the region. This strong resilience shown by the regional economy helps us to explain the impressive variation registered in the efficiency pillar in RCI 2016 compared to 2013. The labour productivity, the GDP per person employed in industry and services in terms of index with the EU20 $=100$, has improved significantly reaching the level of 123.6 in 2014 (i.e. the reference year for RCI 2016) from 112.5 in 2011 (the reference year for RCI 2013). It's worth noting that this upgrading in the efficiency of productive system happened even in presence of an expansion of the person employed ( +4 thousand the absolute increase), the denominator of the labour productivity. The share of population aged 15-24 not in education, employment or training $\left(\mathrm{NEET}^{3}\right)$ in percentage of population aged 15-24 is the lowest among the Italian regions 9.7\% compared with a national average of $24.1 \%$ and an European incidence of $14.2 \%$. As regard to the pillar market size, the net adjusted disposable household income in PPCS per capita with index EU28 $=100$ is the highest among all the other regions and equal to 133 in line with the Ille de France the most competitive region of France and ranked eighth out 263 European regions.

All the other Italian regions show negative scores in all the macro-pillar in both 2016 and 2013 with the exception of Lombardia (ITC4) and Emilia Romagna (ITH5). However, by analysing the percentage variations we discover quite encouraging improvements even in the southern regions. For example Calabria (ITF6), the worst performing Italian region together with Sicilia (ITG1) (235th and 237th, respectively), shows an upgrading $(+5.1 \%)$ exactly where it was in the latest i.e. in the Basic subindex position (217th in 2016 ranking

\footnotetext{
3 The indicator young people neither in employment nor in education and training, abbreviated as NEET, corresponds to the percentage of the population of a given age group and sex who is not employed and not involved in further education or training. The numerator of the indicator refers to persons meeting these two conditions: they are not employed (i.e. unemployed or inactive according to the International Labour Organisation definition); they have not received any education or training in the four weeks preceding the survey. The denominator is the total population of the same age group and sex, excluding the respondents who have not answered the question 'participation to regular education and training'.
} 
Table 20 Fuzzy C-Medoids RCI 2013-2016 clusters

\begin{tabular}{|c|c|c|c|c|c|c|}
\hline \multirow[t]{2}{*}{ Medoid } & \multicolumn{3}{|l|}{2013} & \multicolumn{3}{|l|}{2016} \\
\hline & Basic & Efficiency & Innovation & Basic & Efficiency & Innovation \\
\hline 1 EL42 N. Aigaio & -1.298 & -1.422 & -1.354 & -1.498 & -1.083 & -1.330 \\
\hline 2 BE21 Antwerpen & 0.647 & 0.852 & 0.838 & 0.526 & 0.722 & 0.902 \\
\hline 3 ITF1 Abruzzo & -0.427 & -0.469 & -0.729 & -0.439 & -0.651 & -0.726 \\
\hline 4 DE23 Oberpfalz & 0.485 & 0.414 & 0.426 & 0.601 & 0.387 & 0.460 \\
\hline 5 FR41 Lorraine & 0.030 & -0.094 & -0.051 & 0.054 & 0.081 & 0.008 \\
\hline
\end{tabular}

from 226th in 2013), followed by Puglia (ITF4) with a relative increase of around $4.0 \%$ compared to 2013.

For Calabria (ITF6) the improvement in the basic pillar is due to an increase in the trust towards local public authorities, a reduction in early school leavers rate (from 16.2 to $15.7 \%$ ), an upgrading in reading (from 184.0 in 2012 to 192.0 in 2015) and mathematics (from 186.9 in 2012 to 194.2 in 2015) proficiency scores of lower and upper secondary students, that all in one were able to more than offset the worsening in the duration of civil disputes (from 758.7 days to 846.2) and the widening in the share of NEET (from 35.8 to $38.2 \%$ ). By contrast Puglia shows an improvement in all the indicators explaining the basic pillar, especially in the efficiency of justice wherein the duration of civil disputes has decreased from 951.9 to 798.8 days.

\subsection{Longitudinal Fuzzy C-Medoids Clustering (L-FCMd) RCI 2013-2016 Subindexes}

The best partition according to the $F S$ index (Sect. 3.2) is the 5 clusters partition. The values of the weights $w_{t}$ that optimize 1 are 0.4 and 0.6 for $t=2013,2016$, respectively.

Two clusters have negative values of the subindexes, cluster 1 (medoid EL42) with all subindexes smaller than -1.00 and cluster 3 (medoid ITF1) with the subindexes in the interval $(-0.50 ;-1.00)$; cluster 5 (medoid FR41) has two negative subindexes (Efficiency 2013 and Innovation 2013) and the other positive but all close to 0.00; two clusters have positive values of the subindexes, cluster 4 (medoid DE23) in the interval $(0.20 ; 0.50)$ and cluster 2 (medoid BE21) in the interval $(0.50 ; 1.00)$ (Table 20).

Italian regions Piemonte (ITC1), Liguria (ITC3), Lombardia (ITC4), Trento (ITH2), Friuli Venezia Giulia (ITH4), Emilia Romagna (ITH5) and Lazio (ITI4) are in cluster 5. All the other Italian regions are in cluster 3.

\subsection{Cross Sectional Fuzzy C-Medoids (CS-FCMd-C) RCI 2016 Subindexes with Contiguity Constraints: Regions of Europe}

The NUTS are grouped into 4 geographic areas: Western Europe, Southern Europe, Central Europe and Northern Europe.

- Western Europe - area 1: Austria, Belgium, France, Germany, Ireland, Luxembourg, The Netherlands, United Kingdom

- Southern Europe - area 2: Cyprus, Greece, Italy, Malta, Portugal, Spain 
Table 21 Fuzzy C-Medoids RCI 2016 clusters with geographic area contiguity constraint

\begin{tabular}{lrrrl}
\hline Medoid & \multicolumn{1}{c}{ Basic } & Efficiency & Innovation & \\
\hline 1 CZ06 Jihovýchod & -0.165 & -0.147 & -0.093 & \\
2 ITF2 Molise & -0.530 & -0.657 & -0.885 & Others \\
3 ITI4 Lazio & -0.287 & -0.241 & -0.081 & ITI4 \\
4 NL11 Groningen & 0.405 & 0.445 & 0.464 & \\
\hline
\end{tabular}

Table 22 Fuzzy C-Medoids RCI 2016 with geographic area constraint cluster composition

\begin{tabular}{|c|c|c|c|c|c|c|c|c|c|c|c|c|c|c|}
\hline & AT & $\mathrm{BE}$ & BG & $\mathrm{CZ}$ & $\mathrm{DE}$ & DK & $\mathrm{EE}$ & EL & ES & FI & FR & HR & $\mathrm{HU}$ & IE \\
\hline Cluster 1 & & & & 1 & & & & & & & & & & \\
\hline Cluster 2 & & & 6 & 6 & & & & 13 & 19 & & & 2 & 7 & \\
\hline \multicolumn{15}{|l|}{ Cluster 3} \\
\hline \multirow[t]{3}{*}{ Cluster 4} & 8 & 9 & & & 37 & 5 & 1 & & & 4 & 22 & & & 2 \\
\hline & 8 & 9 & 6 & 7 & 37 & 5 & 1 & 13 & 19 & 4 & 22 & 2 & 7 & 2 \\
\hline & IT & LT & LU & LV & NL & PI & & PT & RO & SE & SI & SK & UK & \\
\hline Cluster 1 & & & & & & & & & & & & & & 1 \\
\hline Cluster 2 & 20 & & & & & 16 & 7 & & 8 & & 2 & 4 & & 110 \\
\hline Cluster 3 & 1 & & & & & & & & & & & & & 1 \\
\hline \multirow[t]{2}{*}{ Cluster 4} & & 1 & 1 & 1 & 11 & & & & & 8 & & & 34 & 144 \\
\hline & 21 & 1 & 1 & 1 & 11 & 16 & 7 & & 8 & 8 & 2 & 4 & 34 & 256 \\
\hline
\end{tabular}

- Central Europe-area 3: Bulgaria, Croatia, Czech Republic, Hungary, Poland, Romania, Slovakia, Slovenia

- Northern Europe — area 4: Denmark, Estonia, Finland, Latvia, Lituania, Sweden

To take into account the geographic area the clustering model has been applied with a contiguity matrix based on the area. The clustering into four groups has been considered according to the FS index (Sect. 3.2). The spatial parameter $\beta$ has been selected in the interval 0.5-4.0 (step 0.5) in such a way that the within cluster spatial autocorrelation measure is maximized (Sect. 3.2). The selected value of $\beta$ is equal to 0.3 .

In the Fuzzy C-Medoids three clusters have negative values of the subindexes and one cluster positive values. In cluster 1 (medoid CZ06) the three subindexes have negative values in the interval $(0.00 ;-0.20)$; in cluster 2 (medoid ITF2) the three subindexes are negative in the interval $(-0,50 ;-1,00)$; in cluster 3 (medoid ITI4) the subindexes Basic and Efficiency are negative in the interval $(-0.20 ;-0.50)$ and the subindex Innovation in the interval $(-0.00 ;-0.20)$; in cluster 4 (medoid NL11) the three subindexes have positive values in the interval $(0.20 ; 0.50)$ (Table 21$)$.

The Italian region Lazio (ITI4) is in cluster 3 (highest value in Italy of the subindex Innovation); all the other regions are in cluster 2. Except Lazio (ITI4) - single in cluster 3 - the Italian regions are in clusters homogeneous with respect to the area.

The region in cluster 1 is CZ06; the regions in cluster 2 are mostly (first three) regions of Spain (ES), Italy (IT) and Poland (PL); the region in cluster 3 is ITI4; the regions in cluster 4 mostly regions of Germany (DE), France (FR) and United Kingdom (UK) (Table 22). 
Table 23 Fuzzy C-Medoids RCI 2016 with geographic area constraint cross-table cluster/area

\begin{tabular}{lccccr}
\hline & Area 1 & Area 2 & Area 3 & Area 4 & \\
\hline Cluster 1 & & & 1 & & 1 \\
Cluster 2 & & 59 & 51 & & 110 \\
Cluster 3 & & 1 & & & 1 \\
Cluster 4 & 124 & & & 20 & 144 \\
& 124 & 60 & 52 & 20 & 256 \\
\hline
\end{tabular}

The cross-table geographic area/cluster presented in Table 23 shows geographic area homogeneity. The regions in areas 2 and 3 (Southern/Central Europe) are in cluster 2; the regions in areas 1 and 4 (Western/Northern Europe) are in cluster 4.

\subsubsection{Comparison with $\mathrm{RCI} 2016$ Clustering Without Constraints}

The comparison with the clusters RCI 2016 without the constraint on the geographic area (Sect. 4.2) shows an upgrade for regions Campania (ITF3) to Sardegna (ITG2); a downgrade for all the other regions. This means that Italian regions in geographic area 2 are in clusters with regions-in the same area-performing better with respect to regions Campania (ITF3) to Sardegna (ITG2) and worst with respect to all the others.

\subsection{Cross Sectional Fuzzy C-Medoids (CS-FCMd-C) RCI 2016 Subindexes with Contiguity Constraints: Level of GDP}

The RCI is obtained as an average of the subindexes Basic, Efficiency and Innovation with weights depending on the stage of development (Tables 4, 6). To take into account the stage of development the clustering models have been applied with a contiguity matrix based on the five levels of development. The clustering into five groups has been considered according to the $F S$ index (Sect. 3.2). The selected value of the spatial parameter $\beta$ is equal to 0.5 (Sect. 3.2). The Fuzzy C-Medoids partition is presented.

In cluster 3 (medoid HU32) the three subindexes have negative values, Basic and Efficiency in the interval $(-0.50 ;-1.00)$ and Innovation smaller than -1.00 ; in clusters 1 (medoid FR21) and 2 (medoid FR81) two subindexes have a small negative values and one a small positive values, Basic in cluster 1 and Innovation in cluster 2; in cluster 4 (medoid NL11) the three subindexes have positive values in the interval $(0.20 ; 0.50)$ and in cluster 5 (medoid UKD1) the subindexes Basic and Efficiency have positive values in the interval $(0.00 ; 0.20)$ and the Innovation subindex in the interval $(0.20 ; 0.50)$ (Table 24).

The Italian regions Abruzzo (ITF1), Molise (ITF2) and Umbria (ITI2) (GDP level 3) are in cluster 2; regions ITC2, Lombardia (ITC4), Bolzano (ITH1), Trento (ITH2), Veneto (ITH3), Emilia Romagna (ITH5) and Lazio (ITI4) (GDP level 5) are in cluster 4; regions Piemonte (ITC1), Liguria (ITC3), Friuli-Venezia Giulia (ITH4), Toscana (ITI1), Marche (ITI3) (GDP level 4) are in cluster 5. All the other Italian regions (Campania (ITF3) to Sardegna (ITG2)) with GDP level 2) are in cluster 3. Italian regions are in clusters homogeneous with respect to the stage of development as measured by the level of GDP.

The regions in cluster 1 are mostly (first three) regions of France (one region FR21); the regions in cluster 2 regions of Spain (ES), France (FR) and United Kingdom (UK); regions 
Table 24 Fuzzy C-Medoids RCI 2016 clusters with GDP contiguity constraint

\begin{tabular}{lrrrl}
\hline Medoid & Basic & Efficiency & Innovation & \\
\hline 1 FR21 Champagne-Ardenne & 0.081 & -0.118 & -0.026 & \\
2 FR81 Languedoc-Roussillon & -0.026 & -0.055 & 0.034 & ITF1, ITF2 \\
& & & & ITI2 (GDP 3) \\
3 HU32 & -0.986 & -0.900 & -1.098 & $\begin{array}{l}\text { ITF3, ITF4, ITF5 } \\
\text { ITF6, ITG1 }\end{array}$ \\
4 NL11 Groningen & & & & ITG2 (GDP 2) \\
& 0.405 & 0.445 & 0.464 & $\begin{array}{l}\text { ITC2, ITC4, ITH1 } \\
\text { 5 UKD1 Cumbria }\end{array}$ \\
& 0.173 & 0.197 & & ITH2, ITH3 \\
& & & & ITH5, ITI4 (GDP 5) \\
& & & & ITC1, ITC3, ITH4 ITI3 (GDP 4) \\
\hline
\end{tabular}

Table 25 Fuzzy C-Medoids RCI 2016 with GDP constraint cluster composition

\begin{tabular}{|c|c|c|c|c|c|c|c|c|c|c|c|c|c|c|}
\hline & AT & $\mathrm{BE}$ & BG & $\mathrm{CZ}$ & DE & DK & $\mathrm{EE}$ & EL & ES & FI & FR & HR & $\mathrm{HU}$ & IE \\
\hline Cluster 1 & & & & & & & & & & & 1 & & & \\
\hline Cluster 2 & 1 & 4 & & 1 & 5 & 1 & & 1 & 7 & & 12 & & & 1 \\
\hline Cluster 3 & & & 6 & 5 & & & 1 & 11 & 5 & & & 2 & 6 & \\
\hline Cluster 4 & 6 & 3 & & 1 & 24 & 3 & & & 3 & 2 & 1 & & & 1 \\
\hline \multirow[t]{3}{*}{ Cluster 5} & 1 & 2 & & & 8 & 1 & & 1 & 4 & 2 & 8 & & 1 & \\
\hline & 8 & 9 & 6 & 7 & 37 & 5 & 1 & 13 & 19 & 4 & 22 & 2 & 7 & 2 \\
\hline & IT & LT & LU & LV & NL & PI & & PT & RO & SE & SI & SK & UK & \\
\hline Cluster 1 & & & & & & & & & & & & & & 1 \\
\hline Cluster 2 & 3 & & & & & 1 & & 1 & & & & 15 & & 53 \\
\hline Cluster 3 & 6 & 1 & & 1 & & 14 & & 5 & 7 & & 1 & 3 & 2 & 76 \\
\hline Cluster 4 & 7 & & 1 & & 6 & & & & 1 & 3 & & 1 & 7 & 70 \\
\hline \multirow[t]{2}{*}{ Cluster 5} & 5 & & & & 5 & 1 & & 1 & & 5 & 1 & & 10 & 56 \\
\hline & 21 & 1 & 1 & 1 & 11 & 16 & & 7 & 8 & 8 & 2 & 4 & 34 & 256 \\
\hline
\end{tabular}

in cluster 3 of Greece (EL), Poland (PL) and Romania (RO); the regions in cluster 4 mostly regions of Germany (DE), Italy (IT) and United Kingdom (UK) and the regions in cluster 5 mostly regions of Germany (DE), France (FR) and United Kingdom (UK) (Table 25).

The cross-table GDP level/cluster shows GDP level homogeneity under constraint on the level of GDP and coherence. The regions with GDP level 1 and 2 are in cluster 3, respectively; the regions with GDP level 3 are mostly in cluster 2, with GDP 4 mostly in cluster 5 and with GDP 5 mostly in cluster 4 (Table 26).

\subsubsection{Comparison with $\mathrm{RCl} 2016$ Clustering Without Constraints}

The comparison with the clusters RCI 2016 without the constraint on the stage of development (Sect. 4.2) shows a slight downgrade for regions with GDP level 2 (in particular with respect to the value of the subindex Innovation), an upgrade for all the other regions. 
Table 26 Fuzzy C-Medoids RCI 2016 with GDP level constraint cross table GDP/area

\begin{tabular}{lcccccr}
\hline & GDP level 1 & GDP level 2 & GDP level 3 & GDP level 4 & GDP level 5 \\
\hline Cluster 1 & & & & 1 & & 1 \\
Cluster 2 & & 1 & 43 & 5 & 4 & 53 \\
Cluster 3 & 19 & 56 & & & 1 & 76 \\
Cluster 4 & & 4 & 6 & 60 & 70 \\
Cluster 5 & & & 6 & 57 & 5 & 56 \\
& 19 & 57 & 53 & 57 & 70 & 256 \\
\hline
\end{tabular}

This means that Italian regions in GDP level 2 are in clusters with regions-with the same GDP level-performing worst in the three subindexes; regions in GDP levels 3, 4 and 5 are in clusters with regions-with the same GDP level-performing better in the three subindexes.

\section{Conclusions}

We presented an application of the Cross Sectional and Longitudinal Fuzzy C-Medoids Clustering with contiguity constraints. The temporal aspect is dealt with by using appropriate measures of dissimilarity between time trajectories, in particular by distinguishing the cross-sectional and Longitudinal (variation over time) aspects of the trajectories. The contiguity among units is dealt with adding a contiguity penalization term to the objective function, depending on a suitable contiguity matrix.

The models are applied to the classification of the European NUTS on the basis of the observed dynamics of the Basic, Efficiency and Innovation subindexes of the Regional Competitiveness Index (RCI) 2013 and 2016. The positioning of the Italian regions is deeply analyzed. Two contiguity constraints, one based on the European Western, Southern, Central and Nothern geographic areas and one on the level of GDP - taken into account in the computation of the RCI-are also introduced in the models.

The application highlights the ability of the proposed models - in particular of the Cross sectional and Longitudinal Fuzzy C-Medoids Clustering with contiguity constraints- to use the information in the multivariate data, either temporal or based on any contiguity relation, allowing a deep study of the characteristics of the Italian regions at the basis of their positioning in the clustering of the European NUTS based on the the subindexes of the RCI.

Possible developments at the application level are the clustering with respect to other composite indexes (Human Development Index, Well Being) and the harmonization of the obtained partitions, besides the extension of the considered periods of time and finest regional levels (an example of application of the RCI at the level NUTS3 is in De Giovanni and Sica (2014)).

Possible developments at the methodological level are the models robust to the presence of outliers. 


\section{References}

Annoni, P., \& Dijkstra, L. (2013). EU Regional Competitiveness Index: RCI 2013, EUR 26060, 168. Luxembourg: Publications Office of the European Union.

Annoni, P., \& Dijkstra, L. (2017). Measuring and monitoring regional competitiveness in the European Union. In R. Huggins \& P. Thompson (Eds.), Handbook of regions and competitiveness - contemporary theories and perspectives on economic development. Edward Elgar Publishing.

Annoni, P., Dijkstra, L., \& Gargano, N. (2017). The EU Regional Competitiveness Index 2016, WP 02/2017, 28. Luxembourg: Publications Office of the European Union.

Bezdek, J. C. (1981). Pattern recognition with fuzzy objective function algorithms. New York: Kluwer.

Caiado, J., Maharaj, E. A., \& D’Urso, P. (2015). Time series clustering. In C. Hennig, M. Meila, F. Murtagh, \& R. Rocci (Eds.), 2006 Handbook of cluster analysis (pp. 241-264). London: Chapman and Hall/ CRC.

Campello, R. J. G. B., \& Hruschka, E. (2006). A fuzzy extension of the silhouette width criterion for cluster analysis. Fuzzy Sets and Systems, 157(21), 2858-2875.

Charron, N., Dijkstra, L., \& Lapuente, V. (2015). Mapping the regional divide in Europe: A measure for assessing quality of government in 206 European Regions. Social Indicators Research, 122, 315-346.

Chuang, K.-S., Tzeng, H.-L., Chen, S., Wu, J., \& Chen, T.-J. (2006). Fuzzy c-means clustering with spatial information for image segmentation. Computerized Medical Imaging and Graphics, 30, 9-15.

Coppi, R., \& D’Urso, P. (2001). The geometric approach to the comparison of multivariate time trajectories. In S. Borra, R. Rocci, M. Vichi, \& M. Schader (Eds.), Advances in data science and classification (pp. 93-100). Heidelberg: Springer.

Coppi, R., \& D’Urso, P. (2002). Fuzzy k-means clustering models for triangular fuzzy time trajectories. Statistical Methods \& Applications, 11, 21-40.

Coppi, R., \& D’Urso, P. (2003). Three-way fuzzy clustering models for LR fuzzy time trajectories. Computational Statistics \& Data Analysis, 43, 149-177.

Coppi, R., \& D’Urso, P. (2006). Fuzzy unsupervised classification of multivariate time trajectories with the Shannon entropy regularization. Computational Statistics \& Data Analysis, 50, 1452-1477.

Coppi, R., D’Urso, P., \& Giordani, P. (2010). A fuzzy clustering model for multivariate spatial time series. Journal of Classification, 27, 54-88.

De Giovanni, L., \& Sica, F. G. M. (2014). Attrattivitá e competitivitá dei territori italiani - I risultati: le dimensioni dell'attrattivitá territoriale (in italian). Rivista di Politica Economica, 4, 115-234.

Delgado-Marquez, B. L., \& García-Velasco, M. (2018). Geographical distribution of the European knowledge base through the lens of a synthetic index. Social Indicators Research, 122, 315-346.

Di Nola, A., Loia, V., \& Staiano, A. (2000). Genetic-based spatial clustering. IEEE International Conference on Fuzzy Systems, 2, 953-956.

D’Urso, P. (2004). Fuzzy C-means clustering models for multivariate time-varying data: Different approaches. International Journal of Uncertainty, Fuzziness and Knowledge-Based Systems, 12, 287-326.

D'Urso, P. (2005). Fuzzy clustering for data time arrays with inlier and outlier time trajectories. IEEE Transactions on Fuzzy Systems, 13, 583-604.

D’Urso, P. (2015). Fuzzy clustering. In C. Hennig, M. Meila, F. Murtagh, \& R. Rocci (Eds.), Handbook of cluster analysis (pp. 545-574). London: Chapman and Hall.

D’Urso, P., Maharaj, E. A., \& Alonso, A. M. (2017a). Fuzzy clustering of time series using extremes. Fuzzy Sets and Systems, 318, 56-79.

D’Urso, P., Massari, R., Cappelli, C., \& De Giovanni, L. (2017b). Autoregressive metric-based trimmed fuzzy clustering with an an application to pm10 time series. Chemometrics and Intelligent Laboratory Systems, 13, 583-604.

European Commission. (2017). The recent reform of the labour market in Italy: A review. In D. Pinelli, R. Torre, L. Pace, L. Cassio, \& A. Arpaia (Eds.), Discussion Paper 072. Luxembourg: Publications Office of the European Union, ISBN 978-92-79-64935-6.

EUROSTAT, Regional Yearbook 2016. (2017). ISBN 978-92-79-71616-4. Luxembourg: Publications Office of the European Union.

Everitt, B., Landau, S., \& Leese, S. (2001). Cluster analysis. London: Arnold Press.

Fouedjio, F. (2016). A hierarchical clustering method for multivariate geostatistical data. Spatial Statistics, $18,333-351$.

Fu, K. S. (1982). Syntactic pattern recognition and applications. San Diego, CA: Academic Press.

García-Escudero, L. A., \& Gordaliza, A. (1999). Robustness properties of k-means and trimmed k-means. Journal of the American Statistical Association, 94(447), 956-969. 
García-Escudero, L. A., Gordaliza, A., Matrán, C., \& Mayo-Iscar, A. (2010). A review of robust clustering methods Adv. Data Analysis and Classification, 4(2), 89-109.

Gittleman, J., \& Kot, M. (1990). Adaptation: Statistics and a null model for estimating phylogenetic effects. Systematic Zoology, 39, 227-241.

Heiser, W. J., \& Groenen, P. J. F. (1997). Cluster differences scaling with a within-clusters loss component and a fuzzy successive approximation strategy to avoid local minima. Psychometrika, 62(1), 63-83.

Hwang, H., Desarbo, W. S., \& Takane, Y. (2007). Fuzzy clusterwise generalized structured component analysis. Psychometrika, 72(2), 181-198.

ISTAT. (2018). Rapporto sulla competitivitá dei settori produttivi, Rome, ISBN 978-88-458-1950-6.

Kaufman, L., \& Rousseeuw, P. (2005). Finding groups in data: An introduction to cluster analysis. New York: Wiley.

Krishnapuram, R., Joshi, A., Nasraoui, O., \& Yi, L. (2001). Low-complexity fuzzy relational clustering algorithms for web mining. IEEE Transactions on Fuzzy Systems, 9(4), 595-607.

Krishnapuram, R., Joshi, A., \& Yi, L. (1999). A fuzzy relative of the k-medoids algorithm with application to web document and snippet clustering. In IEEE international fuzzy systems conference (FUZZIEEE99), Seoul (Vol. 3, pp. 1281-1286).

Kruse, R., Döring, C., \& Lesot, M.-J. (2007). Fundamentals of fuzzy clustering. In J. V. De Oliveira, \& W. Pedrycz (Eds.), Advances in fuzzy clustering \& its applications (pp. 3-30). Chichester: Wiley.

Mazziotta, M., \& Pareto, A. (2018). Use and misuse of PCA for measuring well-being. Social Indicators Research. https://doi.org/10.1007/s11205-018-1933-0.

McBratney, A., \& Moore, A. (1985). Application of fuzzy sets to climatic classification. Agricultural and Forest Meteorology, 35(1-4), 165-185.

OECD. (2008). Handbook on construction composite indicators: Methodology and user guide. France: OECD, ISBN 978-92-64-04345-9.

OECD. (2011). Higher education in regional and city development: Lombardy. France: OECD, ISBN 978-92-64-08946-4.

OECD. (2016). The survey of adult skills: Reader's companion (2nd edn.). France: OECD, ISBN 978-92-64-25807-5.

OECD. (2017). Regions at a Glance 2016. France: OECD, ISBN 978-92-64-25679-8.

Pham, D. L. (2001). Spatial models for fuzzy clustering. Computer Vision and Image Understanding, 84, 285-297.

Sammon, J. W. (1969). A nonlinear mapping for data structure analysis. IEEE Transactions on Computers, C-18, 401-409.

Wedel, M., \& Kamakura, W. A. (1998). Market segmentation: Conceptual and methodological foundations. Dordrecht: Kluwer.

WEF. (2017). The global competitiveness report 2017. Switzerland: WEF, ISBN 978-1-944835-11-8.

Publisher's Note Springer Nature remains neutral with regard to jurisdictional claims in published maps and institutional affiliations. 